\title{
Spin-heat relaxation and thermospin diffusion in atomic Bose and Fermi gases
}

\author{
Clement H. Wong, ${ }^{1}$ H. T. C. Stoof, ${ }^{2}$ and R. A. Duine ${ }^{2}$ \\ ${ }^{1}$ Department of Physics, University of Wisconsin-Madison, Madison, Wisconsin 53706, USA \\ ${ }^{2}$ Institute for Theoretical Physics and Center for Extreme Matter and Emergent Phenomena, Utrecht University, \\ Leuvenlaan 4, 3584 CE Utrecht, Netherlands
}

(Received 3 November 2014; published 2 April 2015)

\begin{abstract}
We study spin-dependent heat transport in quantum gases, focusing on transport phenomena related to pure spin currents and spin-dependent temperatures. Using the Boltzmann equation, we compute the coupled spin-heat transport coefficients as a function of temperature and interaction strength for energy-dependent $s$-wave scattering. We address the issue of whether spin-dependent temperatures can be sustained on a time and length scale relevant for experiments by computing the spin-heat relaxation time and diffusion length. We find that the time scale for spin-heat relaxation time diverges at low temperatures for both bosons and fermions, indicating that the concept of spin-heat accumulation is well defined for degenerate gases. For bosons, we find power-law behavior on approach to Bose condensation above the critical temperature, as expected from the theory of dynamical critical phenomena.
\end{abstract}

DOI: 10.1103/PhysRevA.91.043602 PACS number(s): 67.85.-d, 03.75.-b, 67.10.Fj, 67.10.Jn

\section{INTRODUCTION}

Spin caloritronics is currently an active field of research concerned with studying the spin-dependent generalizations of thermoelectric effects in solid-state materials [1], as well as novel collective effects. Just as the traditional thermoelectric phenomena, i.e., the Seebeck and Peltier effects, have applications in generators, refrigerators, and utilizing waste heat, thermally driven spin currents may have applications in spintronics devices. In fact, the coupling of particle, (pseudo)spin, and heat transport is a general phenomenon not restricted to the solid-state environment. Thermoelectric effects in ultracold atomic gases have recently become a topic of experimental interest [2-5]. In contrast to the multifaceted mechanism of heat transport in the solid state, which includes disorder and phonon scattering, electron-electron interactions, and, in ferromagnetic materials, spin-polarized conductivities, magnon scattering, and spin-flip scattering [6,7], in cold atoms, atom-atom interactions are the only natural source of scattering, which can even be controlled experimentally through Feshbach resonances. Thus, the cold atomic gases provide a clean and controllable environment for studying thermoelectric and spin caloritronic effects at the fundamental level. Conversely, measurements of the spin-heat transport coefficients can be used to extract information about the scattering processes.

In this paper, we consider two-component (pseudo)spin-1/2 atomic gases in a smooth trapping potential, in mechanical equilibrium, where the net forces on the cloud are balanced by the trapping forces [8]. Even for this stationary gas, without any spin polarization, a pure spin current can be established in response to opposite forces on each spin, i.e., a spin force, due to interspin scattering that transfer momentum between opposite spins. This viscosity between spins is called spin drag and has been calculated and measured in Bose and Fermi gases [9-12], and its contribution to the spin diffusion coefficient for electrons, called spin Coulomb drag, has been measured in GaAs quantum wells [13]. Due to the Peltier effect, this spin current is accompanied by a spin-heat current, a difference in the heat currents carried by each spin. The thermodynamic reciprocal effect is the spin-Seebeck effect, by which a spin current is driven by gradients of the spin-heat accumulation, i.e., opposite temperature gradients for the two spin states. This coupling is generic, so that, for example, in the experiment of Ref. [10], spin-dependent heating will occur in the presence of spin currents.

A natural question which arises in considering spindependent heat transport is whether one can, in practice, sustain spin-dependent temperatures, which, in the absence of externally applied spin-dependent heating, will ultimately equilibrate due to interspin scattering. In fact, systems that are modeled with multiple temperatures occur in many subfields in physics, including two-component plasmas with large mass differences [14], magnetic systems excited by femtosecond laser pulses [15], and nanopillar spin valves where the difference between spin-up and spin-down temperatures, called the spin-heat accumulation, and the associated spin-heat relaxation rates and lengths have been measured [16,17]. In this paper, we address this issue specifically for the case of ultracold atomic gases [18]. We show that the spin-heat accumulation can be treated as a quasiequilibrium quantity much like spin accumulation, i.e., spin-dependent chemical potentials, in the presence of spin-flip scattering. We compute the spin-heat relaxation time and length as functions of temperature and interaction strength and find power-law divergences for the relaxation time at degenerate temperatures for both bosons and fermions, indicating that the spin-heat accumulation is, in principle, well defined for degenerate quantum gases. We also find that, depending on the interspin scattering lengths, the relaxation length can be on the order of microns for bosons and millimeters for fermions, which is well within experimental resolution and comparable to or larger than the system size.

Thermally driven spin currents can be utilized in spintronic devices, for example, to move a domain wall [19]. Similarly, the coupled spin-heat transport we study here may be utilized for atomtronic devices that run on spin currents. Therefore, we introduce a dimensionless quantity characterizing spin-heat conversion in this system called $Z_{s} T$ in analogy to the $Z T$ figure of merit that determines the efficiency of solid-state 
thermoelectric devices. We find that for bosons, with strong scattering, $Z_{s} T$ and the spin-Seebeck coefficient are enhanced on approach to the critical temperature of Bose-Einstein condensation, in contrast to the case of weak scattering in Ref. [20]. At weak scattering, we also find a sign change in the spin-Seebeck coefficient.

This paper is organized as follows. In Sec. II, we introduce the Boltzmann and associated hydrodynamic equations for a two-component gas. In Sec. III, we report the results of our calculations of the spin-heat relaxation times and lengths for bosons and fermions. In Sec. IV, we express, in linear response, the spin-dependent response and relaxation coefficients in terms of the collision integral, specializing to the case of unpolarized gases in Sec. IV B. In Sec. V, we develop a moment expansion for the computation of the collision integrals which explicitly preserves Onsager reciprocity. In Sec. VI, we present our results for the transport coefficients as a function of temperature and interaction strength for bosons, extending the work of Ref. [20] to include dependence on scattering length. Relevant thermodynamic properties are summarized in Appendix A, and computation details are given in Appendix B.

\section{SPIN-DEPENDENT BOLTZMANN AND HYDRODYNAMIC EQUATIONS}

We will compute the transport coefficients of the twocomponent gas using the semiclassical Boltzmann equation for the distribution functions $n_{\mathbf{p} \sigma}(\mathbf{r}, t)$, given by

$$
\left(\partial_{t}+\mathbf{v}_{\mathbf{p}} \cdot \nabla+\mathbf{f}_{\sigma} \cdot \nabla_{\mathbf{p}}\right) n_{\mathbf{p} \sigma}(\mathbf{r}, t)=\mathcal{C}_{\mathbf{p} \sigma}\left[n_{+}, n_{-}\right],
$$

where $\sigma= \pm$ label the pseudospin index, $\mathbf{f}_{\sigma}$ are external forces, and

$$
\begin{aligned}
\mathcal{C}_{\mathbf{p}_{1} \sigma}\left[n_{+}, n_{-}\right]= & \frac{2 \pi}{\hbar} \prod_{i=2}^{4} \int \frac{d \mathbf{p}_{i}}{(2 \pi \hbar)^{3}}(2 \pi \hbar)^{3} \delta^{3}\left(\mathbf{p}_{1}+\mathbf{p}_{2}-\mathbf{p}_{3}-\mathbf{p}_{4}\right) \delta\left(\epsilon_{\mathbf{p}_{1}}+\epsilon_{\mathbf{p}_{2}}-\epsilon_{\mathbf{p}_{3}}-\epsilon_{\mathbf{p}_{4}}\right) \\
& \times \sum_{\tau= \pm} W_{\sigma \tau}\left[n_{3 \sigma} n_{4 \tau}\left(1+\zeta n_{1 \sigma}\right)\left(1+\zeta n_{2 \tau}\right)-n_{1 \sigma} n_{2 \tau}\left(1+\zeta n_{3 \sigma}\right)\left(1+\zeta n_{4 \tau}\right)\right]
\end{aligned}
$$

is the collision integral that describes two-body elastic and spin-conserving scattering of particles from the momentum and spin states $\left(\mathbf{p}_{1} \sigma, \mathbf{p}_{2} \tau\right)$ to $\left(\mathbf{p}_{3} \sigma, \mathbf{p}_{4} \tau\right)$, where $\zeta= \pm 1$ pertains to bosons (+) and fermions (-). In Eq. (2), we defined transition probabilities $W_{\sigma \tau}$ that take into account Bose and Fermi particle statistics, given by

$$
\begin{aligned}
& W_{+-}\left(p_{r}, \chi\right)=\left|T_{+-}\left(p_{r}, \chi\right)\right|^{2}=W_{-+}\left(p_{r}, \chi\right), \\
& W_{\sigma \sigma}\left(p_{r}, \chi\right)=\frac{1}{2}\left|T_{\sigma \sigma}\left(p_{r}, \chi\right)+\zeta T_{\sigma \sigma}\left(p_{r}, \pi-\chi\right)\right|^{2},
\end{aligned}
$$

where $T_{\sigma \tau}\left(p_{r}, \chi\right) \equiv\left\langle\mathbf{p}_{r}^{\prime}, \sigma \tau|\hat{T}| \mathbf{p}_{r}, \sigma \tau\right\rangle$ is the two-body transition matrix element between incoming and outgoing relative momenta $\mathbf{p}_{r}=\left(\mathbf{p}_{1}-\mathbf{p}_{2}\right) / 2$ and $\mathbf{p}_{r}^{\prime}=\left(\mathbf{p}_{3}-\mathbf{p}_{4}\right) / 2$, respectively, $p_{r}=\left|\mathbf{p}_{r}\right|$, and $\chi$ is the angle between relative momenta defined by $\cos \chi \equiv \hat{\mathbf{p}}_{r} \cdot \hat{\mathbf{p}}_{r}^{\prime}$, where $\hat{\mathbf{p}}=\mathbf{p} /|\mathbf{p}|$. The transition probabilities in Eq. (3) are related to the differential cross section for scattering between spin $\sigma$ and $\tau$ particles by $d \sigma_{\sigma \tau} / d \Omega=\left(m / 4 \pi \hbar^{2}\right)^{2} W_{\sigma \tau}$. While the formalism we present in the following applies for a generic spin-dependent scattering cross section, we will specifically compute transport coefficients for $s$-wave scattering, which is independent of $\chi$,

$$
\frac{d \sigma_{+-}}{d \Omega}=\frac{a^{2}}{1+\left(p_{r} a / \hbar\right)^{2}},
$$

where $a$ is the interspin $s$-wave scattering length [21]. For bosons, we consider equal interspin and intraspin scattering lengths, so that $d \sigma_{\sigma \sigma} / d \Omega=2 d \sigma_{+-} / d \Omega$.

The hydrodynamic equations for the spin $\sigma$ particle density, momentum, and energy densities given by taking $\int \frac{d \mathbf{p}}{(2 \pi \hbar)^{3}}\left\{1, \mathbf{p}, \epsilon_{\mathbf{p}}\right\}$ times Eq. (1) are, respectively,

$$
\begin{gathered}
\partial_{t} \rho_{\sigma}+\nabla \cdot\left(\rho_{\sigma} \mathbf{v}_{\sigma}\right)=0 \\
m \rho_{\sigma}\left(\partial_{t}+\mathbf{v}_{\sigma} \cdot \nabla\right) \mathbf{v}_{\sigma}-\rho_{\sigma} \mathbf{f}_{\sigma}=-\nabla \cdot \overleftrightarrow{\boldsymbol{\pi}}_{\sigma}+\boldsymbol{\Gamma}_{\sigma}, \\
\partial_{t} e_{\sigma}+\nabla \cdot \mathbf{j}_{e \sigma}=\mathbf{f}_{\sigma} \cdot \mathbf{j}_{\sigma}+\Gamma_{\sigma},
\end{gathered}
$$

where the particle density, particle and energy currents, and average velocity densities are defined by

$$
\left(\begin{array}{c}
\rho_{\sigma} \\
e_{\sigma} \\
\mathbf{j}_{\sigma} \\
\mathbf{j}_{e \sigma}
\end{array}\right) \equiv \int \frac{d \mathbf{p}}{(2 \pi \hbar)^{3}}\left(\begin{array}{c}
1 \\
\epsilon_{\mathbf{p}} \\
\mathbf{v}_{p} \\
\epsilon_{\mathbf{p}} \mathbf{v}_{p}
\end{array}\right) n_{\mathbf{p} \sigma},
$$

where $\mathbf{j}_{\sigma} \equiv \rho_{\sigma} \mathbf{v}_{\sigma}, \mathbf{v}_{p}=\nabla_{\mathbf{p}} \epsilon_{\mathbf{p}}$, the stress tensor is

$$
\pi_{i j \sigma} \equiv \rho_{\sigma} v_{\sigma i} v_{\sigma j}-\frac{1}{m} \int \frac{d \mathbf{p}}{(2 \pi \hbar)^{3}} p_{i} p_{j} n_{\mathbf{p} \sigma},
$$

and we defined

$$
\left(\begin{array}{l}
\Gamma_{\sigma} \\
\Gamma_{\sigma}
\end{array}\right)=\int \frac{d \mathbf{p}}{(2 \pi \hbar)^{3}}\left(\begin{array}{c}
\epsilon_{\mathbf{p}} \\
\mathbf{p}
\end{array}\right) \mathcal{C}_{\mathbf{p} \sigma}[\vec{n}]
$$

where $\vec{n}_{\mathbf{p}}=\left(n_{\mathbf{p}+}, n_{\mathbf{p}-}\right)$; henceforth an arrow denotes vectors in spin space. These source terms represent the transfer of energy and momentum through interspin scattering and are proportional to the spin drag and spin-dependent temperature relaxation rates, which are the focus of this work.

We note here that the spin $\sigma$ collision integrals and their sum possess collisional invariants corresponding to conservation laws. The particle continuity equation (4) reflects the conservation of the spin $\sigma$ particle number in the absence of spin-flip scattering, so that

$$
\int \frac{d \mathbf{p}}{(2 \pi \hbar)^{3}} \mathcal{C}_{\mathbf{p} \sigma}[\vec{n}]=0,
$$


and furthermore, since the total energy and momentum are conserved, we must have

$$
\sum_{\sigma}\left(\begin{array}{l}
\Gamma_{\sigma}[\vec{n}] \\
\Gamma_{\sigma}[\vec{n}]
\end{array}\right)=0 .
$$

These identities will be used in subsequent sections.

Since we are interested in heating, we transform the energy equation (6) into an entropy-production equation following standard fluid mechanics [22], and we find

$$
\rho_{\sigma} T_{\sigma}\left(\partial_{t}+\mathbf{v}_{\sigma} \cdot \nabla\right) s_{\sigma}=\mathbf{f}_{\sigma} \cdot \mathbf{j}_{\sigma}-\nabla \cdot \mathbf{q}_{\sigma}+\Gamma_{\sigma}+\mathbf{v}_{\sigma} \cdot \boldsymbol{\Gamma}_{\sigma},
$$

where $T_{\sigma}$ and $s_{\sigma}$ are the spin $\sigma$ temperature and entropy per particle, respectively, and we define the heat current by

$$
\mathbf{q}_{\sigma}=\mathbf{j}_{e \sigma}-\rho_{\sigma} \mathbf{v}_{\sigma}\left(\frac{m v_{\sigma}^{2}}{2}+w_{\sigma}\right) .
$$

This definition subtracts the spin $\sigma$ energy current, the second term in the right-hand side of Eq. (11), which contains the enthalpy per particle $w_{\sigma}$, related to the energy per particle $\epsilon_{\sigma}=$ $e_{\sigma} / \rho_{\sigma}$ by $w_{\sigma}=\epsilon_{\sigma}+p_{\sigma} / \rho_{\sigma}$. Thus, the energy flux through the surface of a fluid element includes the work done by pressure forces $\oint p_{\sigma} \mathbf{v}_{\sigma} \cdot d \mathbf{S}$, with $d \mathbf{S}$ being the normal vector surface, which should be subtracted to obtain the heat current $[22,23]$. It is also readily verified that this is the energy flux defined in Eq. (7) for a rigid shift $f_{\mathbf{p} \sigma} \rightarrow f_{\mathbf{p}-m \mathbf{v}_{\sigma}, \sigma}$ of the local Bose or Fermi distribution.

\section{SPIN-HEAT RELAXATION LENGTH AND TIME}

In this section, we use semiphenomenological arguments to deduce the form of the spin-dependent temperature diffusion equations, which will define the spin-heat relaxation length and time, $\lambda_{\text {st }}$ and $\tau_{\text {st }}$, respectively. We then report our results on the temperature and interaction strength dependence of these coefficients based on the solution of the Boltzmann equations (1). Microscopic expressions for these coefficients are given in Sec. IV B 1.

We first transform Eqs. (10) into temperature diffusion equations, again following standard fluid mechanics [22] but keeping track of the heat exchanges between spins. We express the left-hand side of Eqs. (10), which represents the heat gained by spin $\sigma$ particles in a fluid element per unit volume per unit time, in terms of temperature derivatives as $\rho_{\sigma} c_{p}^{\sigma}\left(\partial_{t}+\right.$ $\left.\mathbf{v}_{\sigma} \cdot \nabla\right) T_{\sigma}$, where $c_{p}^{\sigma}=T_{\sigma}\left(\partial s / \partial T_{\sigma}\right)_{p}$ is the heat capacity per particle at constant pressure, and we assume linear-response heat currents $\mathbf{q}_{\sigma}=-\sum_{\tau= \pm} \kappa_{\sigma \tau}^{\prime}(T) \nabla T_{\tau}$, where $\kappa_{\sigma \tau}^{\prime}(T)$ are the spin-dependent heat conductivities. Then, for the case of zero external forces $\left(\mathbf{f}_{\sigma}=0\right)$, equal densities $\rho_{+}=\rho_{-}=\rho$, and zero total particle current $\mathbf{v}_{+}+\mathbf{v}_{-}=0$, Eq. (10) becomes

$$
\rho c_{p} \partial_{t} T_{\sigma}=\sum_{\tau= \pm} \nabla \cdot\left(\kappa_{\sigma \tau}^{\prime} \nabla T_{\tau}\right)+\sigma \Gamma_{s},
$$

where on the left-hand side we kept terms to leading order in the spin-heat accumulation $T_{s}=T_{+}-T_{-}$and we defined $\Gamma_{s}=\Gamma_{+}-\Gamma_{-}$. Since $\Gamma_{s}$ is a relaxation term for $T_{s}, \Gamma_{s}=0$ when $T_{s}=0$, so that in linear response, it can be expanded as $\Gamma_{s}=-\rho c_{p} T_{s} / \tau_{\mathrm{st}}$, with $\tau_{\mathrm{st}}$ being the spin-heat relaxation time [24]. Taking the difference of the spin-up and spin-down

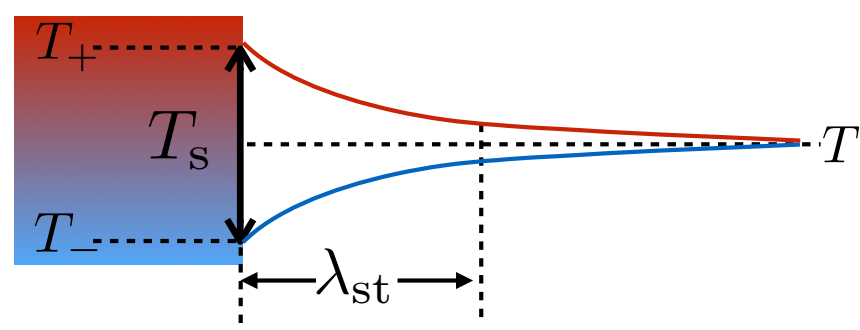

FIG. 1. (Color online) Illustration of spin-dependent temperature gradients that decay on a length scale characterized by the spin-heat relaxation length $\lambda_{\text {st }}$.

components of Eq. (12) and specializing to the case of opposite temperature gradients, $\nabla T_{+}=-\nabla T_{-}$, the spin-heat diffusion equation reads

$$
\partial_{t} T_{s}=\frac{\kappa_{s}^{\prime}}{\rho c_{p}} \nabla^{2} T_{s}-\frac{T_{s}}{\tau_{\mathrm{st}}},
$$

where $\kappa_{s}=\kappa_{++}-\kappa_{+-}$is the spin-heat conductivity [25]. In a steady state, the spin-heat diffusion length that sets the length on which $T_{s} \neq 0$ is given by $\lambda_{s}=\sqrt{\kappa_{s}^{\prime} \tau_{s} / \rho c_{p}}$. Such a steady state could be accomplished in practice, for example, by a laser spin-selectively heating one side of the atomic cloud. Such a situation approximated by the boundary condition of a fixed $T_{s}$ on the left side, with $T_{s}$ penetrating to a depth of $\lambda_{\text {st }}$, is illustrated in Fig. 1.

In Fig. 2, we plot the normalized spin-heat relaxations rates and lengths, $\hbar / k_{B} T_{c} \tau_{\mathrm{st}}$ and $\lambda_{\mathrm{st}} / \lambda_{B}$ as a function of $\left(T-T_{c}\right) / T_{c}$ for bosons and $\hbar / k_{B} T_{F} \tau_{\mathrm{st}}$ and $\lambda_{\mathrm{st}} / \lambda_{F}$ as a function of $T / T_{F}$ for fermions, where $T_{c}$ is the temperature of Bose-Einstein condensation and $T_{F}$ is the Fermi temperature. Here, we define $\lambda_{B}=\rho^{-1 / 3}$ for bosons, $\lambda_{F}=2 \pi\left(6 \pi^{2} \rho\right)^{-1 / 3}$ is the Fermi wavelength for fermions, and $\rho$ is the equilibrium density. The power-law dependence of the spin-heat relaxation coefficients on $T-T_{c}$ and $T / T_{F}$ is evident in the logarithmic plots. These plots also suggest power-law behavior as a function of interspin scattering lengths for $a / \lambda_{B} \leqslant 1$ and $a / \lambda_{F} \leqslant 1$.

For fermions, the spin-heat relaxation time $\left(\tau_{\mathrm{st}}\right)$ and length ( $\left.\lambda_{\text {st }}\right)$ diverge as $T / T_{F} \rightarrow 0$. This behavior is expected from Pauli blocking, the suppression of scattering due to Fermi statistics. Figure 2(b) shows relaxation lengths $\lambda_{\text {st }}$ from $10 \lambda_{F}$ up to $10^{5} \lambda_{F}$. For a typical density of $\rho=10^{12} \mathrm{~cm}^{-3}$, we have $\lambda_{F} \sim \lambda_{B} \sim 1 \mu \mathrm{m}$, which gives $\lambda_{\mathrm{st}} \sim 10^{-2}-10^{2} \mathrm{~mm}$, well within experimental resolution and much longer than spin-heat relaxation lengths found in the solid state $[16,26]$. We note that at $T=2 T_{F}$, one enters the high-temperature limit of a classical two-component gas, and from Fig. 2(b), one still finds $\lambda_{\text {st }} \geqslant 0.1 \mu \mathrm{m}$ in this regime.

For bosons, the relaxation time also exhibits a powerlaw divergence as $T \rightarrow T_{c}$, as shown Fig. 2(c), while the relaxation length $\lambda_{\mathrm{st}}$ remains finite. Figure 2(d) shows that at $\left(T-T_{c}\right) / T_{c} \simeq 0.1$ and $\lambda_{B}=1 \mu \mathrm{m}$, we have $\lambda_{\mathrm{st}} \sim 1-10^{2} \mu \mathrm{m}$ for $a / \lambda_{B} \sim 10^{-1}-10^{-2}$ and $\lambda_{\mathrm{st}} \sim 10^{-1}-10^{-2} \mu \mathrm{m}$ for $a / \lambda_{B} \sim 1-10$. Thus, for weak scattering $a / \lambda_{B}<1, \lambda_{\text {st }}$ is within experimental resolution [27].

The physical interpretation for the behavior of the bosonic relaxation coefficients is less obvious. At degenerate temperatures, one expects bosonic enhancement of scattering 

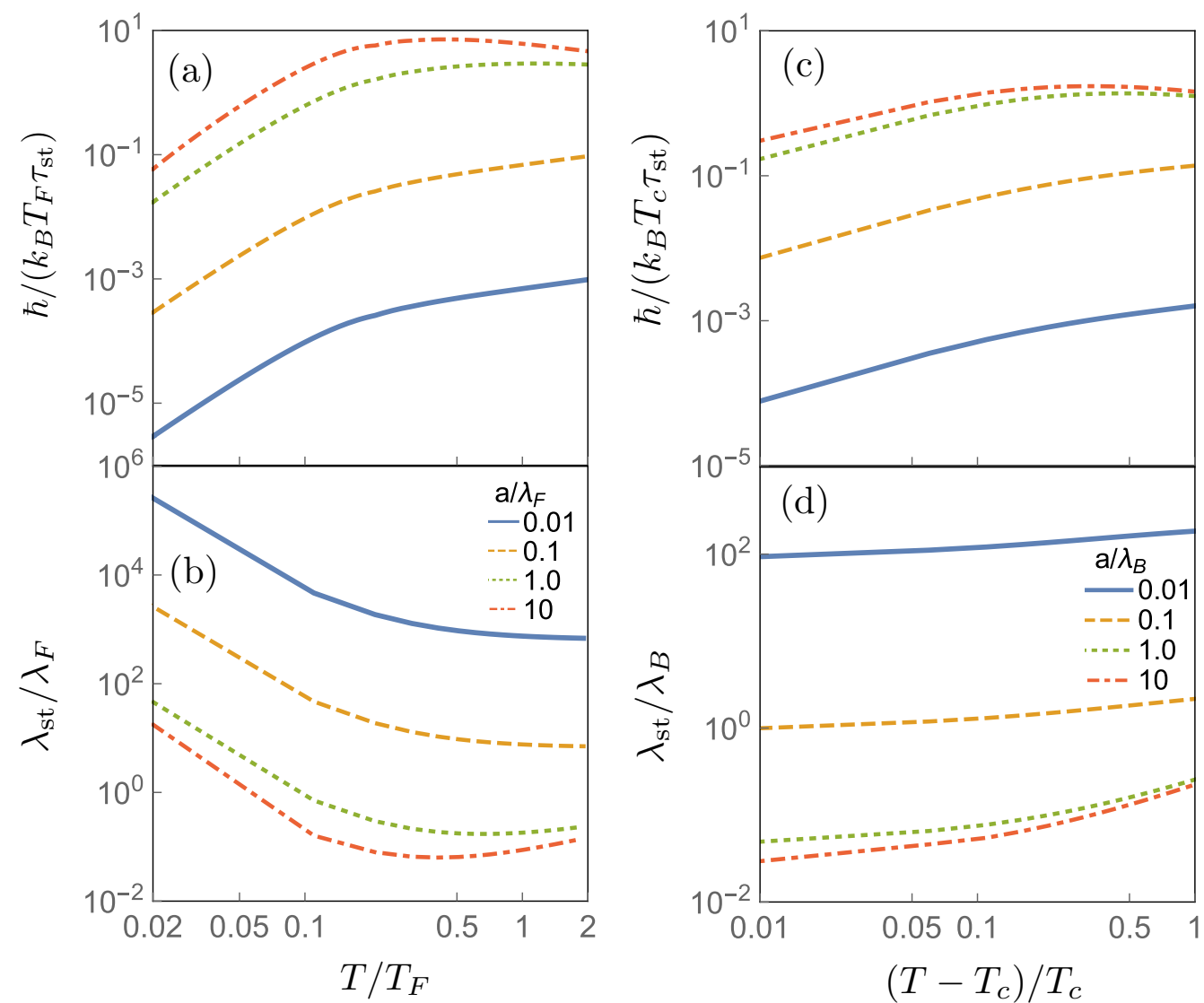

FIG. 2. (Color online) (left) Log-log plots of the normalized spin-heat relaxation rate and length for fermions, (a) $\hbar / k_{B} T_{F} \tau_{\mathrm{st}}$ and (b) $\lambda_{\mathrm{st}} / \lambda_{F}$, respectively, for the interspin scattering lengths $a / \lambda_{F}=(0.01,0.1,1,10)$. (right) Log-log plots of the normalized spin-heat relaxation rate and length for bosons, (c) $\hbar / k_{B} T_{c} \tau_{\mathrm{st}}$ and (d) $\lambda_{\mathrm{st}} / \lambda_{B}$, respectively, for the interspin scattering lengths $a / \lambda_{B}=(0.01,0.1,1,10)$.

to be important. This effect, for example, causes the spindrag relaxation time to vanish as $T \rightarrow T_{c}$ [28,29]. However, mathematically, the divergence in the heat capacity $c_{p}$ as $T \rightarrow$ $T_{c}$ dominates over the Bose enhancement of the relaxation integral $\Gamma_{s}$, causing the relaxation length $\tau_{\text {st }}$ to diverge. Physically, the diverging heat capacity indicates that an increasing amount of heat is necessary per temperature change as $T \rightarrow T_{c}$, which stabilizes the spin-heat accumulation. On the other hand, the relaxation length $\lambda_{\text {st }} \sim \sqrt{\kappa_{s}^{\prime} / \Gamma_{s}}$ decreases as $T \rightarrow T_{c}$ because of Bose enhancement, but for weak scattering this enhancement is weak enough that $\lambda_{\text {st }}$ remains finite even near $T_{c}$.

In the remainder of the paper, we present a detailed computation of the spin-heat relaxation coefficients and the related spin-heat transport coefficients.

\section{LINEARIZED BOLTZMANN EQUATIONS}

In this section, we will use the linearized Boltzmann equation to derive microscopic expressions for spin-dependent particle and heat transport and relaxation coefficients, in linear response to perturbations from equilibrium, i.e., gradients in temperature and density, and external forces. We will outline a general method for obtaining the linear-response coefficients for generic spin-dependent forces and clarify the relation between our work and that of Ref. [30] and the corresponding classical problem in the literature [31]. We then specialize to the response to spin-force and spin-heat accumulation gradients, which is the focus of this work.

We will employ the standard Chapman-Enskog expansion of the nonequilibrium distribution [32],

$$
\begin{aligned}
n_{\mathbf{p} \sigma}(\mathbf{r}, t) & =f_{\mathbf{p} \sigma}(\mathbf{r}, t)-\partial_{\epsilon} f_{\mathbf{p} \sigma}^{0} \Phi_{\mathbf{p} \sigma}(\mathbf{r}) ; \\
f_{\mathbf{p} \sigma}^{0} & =\frac{1}{\exp \left[\left(\epsilon_{\mathbf{p}}-\mu_{\sigma}\right) / k_{B} T\right]-\zeta}, \\
\partial_{\epsilon} f_{\mathbf{p} \sigma}^{0} & =-\frac{f_{\mathbf{p} \sigma}^{0}\left(1+\zeta f_{\mathbf{p} \sigma}^{0}\right)}{k_{B} T}, \\
f_{\mathbf{p} \sigma}(\mathbf{r}, t) & =\frac{1}{\exp \left\{\left[\epsilon_{\mathbf{p}}-\mu_{\sigma}(\mathbf{r}, t)\right] / k_{B} T_{\sigma}(\mathbf{r}, t)\right\}-\zeta},
\end{aligned}
$$

where $\epsilon_{\mathbf{p}}=\mathbf{p}^{2} / 2 m$ is the free-particle dispersion, $f_{\mathbf{p} \sigma}^{0}$ and $f_{\mathbf{p} \sigma}(\mathbf{r}, t)$ are the global and local equilibrium Bose (Fermi) distributions, respectively, $\mu_{\sigma}(\mathbf{r}, t)$ and $T_{\sigma}(\mathbf{r}, t)$ are the local, spin-dependent chemical potentials and temperatures which are determined by the local particle and energy densities (see Appendix A), and the perturbed distribution $\Phi_{\mathbf{p} \sigma}(\mathbf{r})$ describes the response to spatial inhomogeneities. The parametrization of the distribution function in Eq. (14) represents the leadingorder expansion in the ratio of the mean free path to spatial gradients, called the Knudsen number [33], which is assumed 
to be small in our perturbative solution, to be given in following sections.

\section{A. Response to generic spin-dependent forces}

Substituting the expansion (14) in Eq. (1), the Boltzmann equation separates into an equation of order $\nabla$ and one of $\left(\partial_{t}, \nabla^{2}\right)$. To leading order in $T_{s}$, they are

$$
\begin{gathered}
\frac{w_{\sigma}-\epsilon_{\mathbf{p}}}{T}\left(\mathbf{v}_{\mathbf{p}} \cdot \nabla\right) T_{\sigma}+\mathbf{v}_{p} \cdot \mathbf{F}_{\sigma}=\frac{\mathcal{C}_{\mathbf{p} \sigma}[\vec{\Phi}(\mathbf{r})]}{\partial_{\epsilon} f_{\mathbf{p} \sigma}^{0}}, \\
\frac{w_{\sigma}-\epsilon_{\mathbf{p}}}{T} \partial_{t} T_{\sigma}+\left(\mathbf{v}_{\mathbf{p}} \cdot \nabla+\mathbf{f}_{\sigma} \cdot \nabla_{\mathbf{p}}\right) \Phi_{\mathbf{p} \sigma}(\mathbf{r})=\frac{\mathcal{C}_{\mathbf{p} \sigma}[\vec{f}(\mathbf{r}, t)]}{\partial_{\epsilon} f_{\mathbf{p} \sigma}^{0}},
\end{gathered}
$$

where $w_{\sigma}$ is the enthalpy per particle and we introduced the spin $\sigma$ thermodynamic force $\mathbf{F}_{\sigma}=\mathbf{f}_{\sigma}-\nabla p_{\sigma} / \rho_{\sigma}$. Following standard conventions [33], in the advective terms in the left-hand side of Eq. (15), we choose pressure and temperature as our independent variables, with changes in the chemical potential $\mu_{\sigma}=\mu_{\sigma}\left(p_{\sigma}, T_{\sigma}\right)$ given by the Gibbs-Duhem relation,

$$
d \mu_{\sigma}=-s_{\sigma} d T_{\sigma}+d p_{\sigma} / \rho_{\sigma}
$$

where $s_{\sigma}$ and $p_{\sigma}$ are the spin $\sigma$ entropy per particle and pressure, respectively. We then eliminated $\mu_{\sigma}$ in favor of $w_{\sigma}$ using the thermodynamic identity $w_{\sigma}=\mu_{\sigma}+T s_{\sigma}$. Appendix A summarizes some thermodynamic properties of the equilibrium gas.

Linearizing the collision integral in Eq. (15) with respect to $\Phi_{\mathbf{p} \sigma}$ and performing the integrations over final momenta $\mathbf{p}_{3}$ and $\mathbf{p}_{4}$, we have

$$
\begin{aligned}
\mathcal{C}_{\mathbf{p}_{1} \sigma}[\vec{\Phi}]= & -f_{\mathbf{p}_{1} \sigma}^{0} \int \frac{d \mathbf{p}_{2}}{(2 \pi \hbar)^{3}}\left|\mathbf{v}_{r}\right| \int d \Omega_{r}^{\prime} \sum_{\tau= \pm} \frac{d \sigma_{\sigma \tau}}{d \Omega_{r}^{\prime}} \\
& \times f_{\mathbf{p}_{2} \tau}^{0}\left(1+\zeta f_{\mathbf{p}_{3} \sigma}^{0}\right)\left(1+\zeta f_{\mathbf{p}_{4} \tau}^{0}\right) \\
& \times\left(\Phi_{\mathbf{p}_{3} \sigma}+\Phi_{\mathbf{p}_{4} \tau}-\Phi_{\mathbf{p}_{1} \sigma}-\Phi_{\mathbf{p}_{2} \tau}\right),
\end{aligned}
$$

where $\mathbf{v}_{r}=2 \mathbf{p}_{r} / m$ is the relative velocity [34] and $\Omega_{r}^{\prime}$ are the spherical angles of $\hat{\mathbf{p}}_{r}^{\prime}$. In the integrand of Eq. (17), energy and momentum conservation has been enforced, so that $\mathbf{p}_{3}=$ $\mathbf{P} / 2+\mathbf{p}_{r}^{\prime}, \mathbf{p}_{4}=\mathbf{P} / 2-\mathbf{p}_{r}^{\prime}$, where $\mathbf{P}=\mathbf{p}_{1}+\mathbf{p}_{2}$ is the centerof-mass momentum and $\left|\mathbf{p}_{r}\right|=\left|\mathbf{p}_{r}^{\prime}\right|$ is the relative momentum. We note that this linearized collisional possesses the same collisional invariants given in Eq. (9).

Following the standard approach for solving the Boltzmann equation [35], we first solve Eqs. (15) and (17) for the perturbed distributions $\Phi_{\mathbf{p} \sigma}$ and then substitute them into Eq. (16). At the level of the hydrodynamic equations, this procedure corresponds to substituting the linear response currents due to $\Phi_{\mathbf{p} \sigma}$ into the continuity equations, Eqs. (4), (5), and (6), resulting in diffusion equations that govern the spatialtemporal dependence of $T_{\sigma}$ and $\mathbf{F}_{\sigma}$. The collision integral in Eq. (16) leads to the spin-heat relaxation term $\Gamma_{\sigma}$ and is nonzero only for the nonequilibrium part $f_{\mathbf{p} \sigma}(t)$ due to the spin-heat accumulation $T_{s}$. In Sec. IV B 1, we will expand this term to leading order in $T_{s}$ to compute the relaxation time. We note that Eq. (15) does not contribute to the energy continuity equation since $\int d \mathbf{p} \epsilon_{\mathbf{p}}$ times Eq. (20) equals zero because the left-hand side vanishes by isotropy and $\Gamma_{\sigma}\left[\partial_{\epsilon} f \Phi_{\mathbf{p} \sigma}\right]=0$.
To solve Eq. (15), we parametrize the perturbed distribution in linear response as

$$
\begin{aligned}
\Phi_{\mathbf{p} \sigma} & =\sum_{\tau} \boldsymbol{\Phi}_{F}^{\sigma \tau}(\mathbf{p}) \cdot \mathbf{F}_{\tau}+\boldsymbol{\Phi}_{T}^{\sigma \tau}(\mathbf{p}) \cdot\left(-k_{B} \nabla T_{\tau}\right) \\
& \equiv \sum_{\alpha, \tau} \boldsymbol{\Phi}_{\alpha}^{\sigma \tau} \cdot \mathbf{X}_{\alpha \tau},
\end{aligned}
$$

where $\alpha=F, T$ labels the thermodynamic forces $\mathbf{X}_{F \sigma}=\mathbf{F}_{\sigma}$ and $\mathbf{X}_{T \sigma}=-k_{B} \nabla T_{\sigma}$. By symmetry, we have $\boldsymbol{\Phi}_{\alpha}^{+-}=\boldsymbol{\Phi}_{\alpha}^{-+}$. Substituting Eq. (18) into the linearized collision integral (17), we have

$$
\mathcal{C}_{\mathbf{p} \sigma}[\vec{\phi}]=\sum_{\alpha, \tau} \mathcal{C}_{\mathbf{p} \alpha}^{\sigma \tau} \cdot \mathbf{X}_{\alpha \tau}, \quad \mathcal{C}_{\mathbf{p} \alpha}^{\sigma \tau} \equiv \mathcal{C}_{\mathbf{p} \sigma}\left[\boldsymbol{\Phi}_{\alpha}^{+\tau}, \boldsymbol{\Phi}_{\alpha}^{-\tau}\right] .
$$

Noting that $\mathbf{F}_{\sigma}$ and $\nabla T_{\sigma}$ are linearly independent, Eq. (15) separates into two equations,

$$
\begin{gathered}
\mathbf{v}_{\mathbf{p}} \cdot \mathbf{F}_{\sigma}=\sum_{\tau} \frac{\mathcal{C}_{\mathbf{p} F}^{\sigma \tau}}{\partial_{\epsilon} f_{\mathbf{p} \sigma}^{0}} \cdot \mathbf{F}_{\tau}, \\
\left(\frac{\epsilon_{\mathbf{p}}-w_{\sigma}}{k_{B} T}\right) \mathbf{v}_{\mathbf{p}} \cdot \nabla T_{\sigma}=\sum_{\tau} \frac{\mathcal{C}_{\mathbf{p} T}^{\sigma \tau}}{\partial_{\epsilon} f_{\mathbf{p} \sigma}^{0}} \cdot \nabla T_{\tau} .
\end{gathered}
$$

Before solving Eq. (20) for $\Phi_{\mathbf{p} \sigma}$ using the method described in Sec. V, we consider the structure of the response coefficients that follow from them. The particle and heat currents, $\mathbf{j}_{\sigma}$ and $\mathbf{q}_{\sigma}$, respectively, are given by

$$
\begin{aligned}
& \left(\begin{array}{l}
\mathbf{j}_{\sigma} \\
\mathbf{q}_{\sigma}
\end{array}\right) \equiv\left\langle\left(\begin{array}{c}
1 \\
\epsilon_{\mathbf{p}}-w_{\sigma}
\end{array}\right) \mathbf{v}_{\mathbf{p}} \Phi_{\mathbf{p} \sigma}\right\rangle \\
& =\sum_{\tau}\left(\begin{array}{cc}
\mathcal{L}_{F F}^{\sigma \tau} & \mathcal{L}_{F T}^{\sigma \tau} \\
k_{B} T \mathcal{L}_{T F}^{\sigma \tau} & k_{B} T \mathcal{L}_{T T}^{\sigma \tau}
\end{array}\right)\left(\begin{array}{c}
\mathbf{F}_{\tau} \\
-k_{B} \nabla T_{\tau}
\end{array}\right) \\
& =\sum_{\beta \tau}\left(\begin{array}{c}
\mathcal{L}_{F \beta}^{\sigma \tau} \\
k_{B} T \mathcal{L}_{T \beta}^{\sigma \tau}
\end{array}\right) \mathbf{X}_{\beta \tau},
\end{aligned}
$$

where we defined a set of response coefficients $\mathcal{L}_{\alpha \beta}^{\sigma \tau}$ with appropriate factors of $T$ taken out for convenience. Defining the momentum-space inner product

$$
\left\langle\Phi_{\sigma} \chi_{\sigma}\right\rangle \equiv-\int \frac{d^{3} p}{(2 \pi \hbar)^{3}} \partial_{\epsilon} f_{\mathbf{p} \sigma}^{0} \Phi_{\mathbf{p} \sigma} \chi_{\mathbf{p} \sigma},
$$

for a generic spin- and momentum-dependent function $\chi_{\mathbf{p} \sigma}$, the response coefficients are given by

$$
\mathcal{L}_{F \beta}^{\sigma \tau}=\left\langle\mathbf{v}_{\mathbf{p}} \otimes \boldsymbol{\Phi}_{\beta}^{\sigma \tau}\right\rangle, \quad \mathcal{L}_{T \beta}^{\sigma \tau}=\left\langle\frac{\epsilon-w_{\sigma}}{k_{B} T} \mathbf{v}_{\mathbf{p}} \otimes \boldsymbol{\Phi}_{\beta}^{\sigma \tau}\right\rangle,
$$

where $\otimes$ denotes the vector tensor product, although $\mathcal{L}_{\alpha \beta}$ will be diagonal in real space since we do not consider any Hall effects in this paper. We note that $\mathcal{L}_{\alpha \beta}^{+-}=\mathcal{L}_{\alpha \beta}^{-+}$in the cases we study here (without time-reversal symmetry breaking), so we have only three independent spin-resolved coefficients. Onsager reciprocity, which we will prove below, also requires these coefficients to be symmetric in the space of thermodynamic forces; hence, $\mathcal{L}_{F T}^{\sigma \tau}=\mathcal{L}_{T F}^{\sigma \tau}$.

Since we are interested in the spin response, we next transform the response matrix to the total particle $(t)$ and spin $(s)$ sectors. Although, in general, the matrix of linear response coefficients has couplings in the $4 \times 4$ space of spins 
and thermodynamic forces, we will consider the case in which the spin and total particle response decouple. Consider the response of the total (j) and spin $\left(\mathbf{j}_{s}\right)$ currents (in units of $\hbar / 2$ ) in response to average $(\mathbf{F})$ and $\operatorname{spin}\left(\mathbf{F}_{s}\right)$ forces defined by $\mathbf{F}_{\sigma}=\mathbf{F}+\sigma \mathbf{F}_{s} / 2$, given by

$$
\begin{aligned}
\left(\begin{array}{l}
\mathbf{j}_{t} \\
\mathbf{j}_{s}
\end{array}\right) & =\left(\begin{array}{c}
\mathbf{j}_{+}+\mathbf{j}_{-} \\
\mathbf{j}_{+}-\mathbf{j}_{-}
\end{array}\right)=\left(\begin{array}{cc}
\mathcal{L}_{F F}^{(t)} & \mathcal{L}_{F F}^{(s t)} / 2 \\
\mathcal{L}_{F F}^{(s t)} & \mathcal{L}_{F F}^{(s)}
\end{array}\right)\left(\begin{array}{c}
\mathbf{F} \\
\mathbf{F}_{s}
\end{array}\right) ; \\
\mathcal{L}_{F F}^{(t)} & =\mathcal{L}_{F F}^{++}+\mathcal{L}_{F F}^{--}+2 \mathcal{L}_{F F}^{+-}, \\
\mathcal{L}_{F F}^{(t s)} & =\mathcal{L}_{F F}^{++}-\mathcal{L}_{F F}^{--}, \\
\mathcal{L}_{F F}^{(s)} & =\frac{\mathcal{L}_{F F}^{++}+\mathcal{L}_{F F}^{--}}{2}-\mathcal{L}_{F F}^{+-},
\end{aligned}
$$

and the analogous response matrix holds for the response $\mathcal{L}_{T T}^{\sigma \tau}$ and cross response $\mathcal{L}_{F T}^{\sigma \tau}$. We note that our spin current in general differs from the relative current sometimes defined in the literature of two-component gases [31],

$$
\mathbf{j}_{\mathrm{rel}}=\mathbf{j}_{s}-\left(\rho_{s} / \rho\right) \mathbf{j}, \quad \rho_{s}=\rho_{+}-\rho_{-},
$$

which subtracts the spin current carried by the average velocity of the fluid when it is spin polarized, although in the unpolarized case studied below they are equal. Our definition of the spin current has the advantage that it is the current that couples to spin-dependent potentials in the Hamiltonian, which makes it convenient for comparison with calculations using the Kubo formula.

The spin and total particle response decouples when the intraspin response coefficients are equal, $\mathcal{L}_{\alpha \beta}^{++}=\mathcal{L}_{\alpha \beta}^{--}$, so that $\mathcal{L}_{\alpha \beta}^{(s t)}=0$, which means that the center-of-mass motion of the atomic cloud is decoupled from the relative motion of its components, i.e., the spin currents. In this case, the remaining two independent spin-resolved coefficients, given by $\mathcal{L}_{\alpha \beta}^{(t)}=$ $2\left(\mathcal{L}_{\alpha \beta}^{++}+\mathcal{L}_{\alpha \beta}^{+-}\right)$and $\mathcal{L}_{\alpha \beta}^{(s)}=\mathcal{L}_{\alpha \beta}^{++}-\mathcal{L}_{\alpha \beta}^{+-}$, can be determined by the response for two cases: (i) when the average components are zero, $\nabla T_{+}=-\nabla T_{-}$and $\mathbf{F}_{+}=-\mathbf{F}_{-}$, and (ii) when the spin components are zero, $\nabla T_{+}=\nabla T_{-}$and $\mathbf{F}_{+}=\mathbf{F}_{-}$. The latter was recently studied in the high-temperature limit in Ref. [30], where the "spin-Seebeck" coefficient is proportional to $\mathcal{L}_{F T}^{(s t)}$. In the next section, we will consider the former case of opposite forces, which drives pure spin currents stabilized by interspin scattering.

\section{B. Response in an unpolarized gas}

Henceforth, we consider the case of equal equilibrium densities (and masses), $\rho_{+}=\rho_{-} \equiv \rho$, and a local-equilibrium distribution with a spin-heat accumulation gradient $T_{s}(\mathbf{r}, t)$, as shown in Fig. 1. Furthermore, we consider equal intraspin scattering cross sections $d \sigma_{++} / d \Omega=d \sigma_{--} / d \Omega$. Then, by symmetry, $\mathcal{L}_{\alpha \beta}^{++}=\mathcal{L}_{\alpha \beta}^{--}$, so that the off-diagonal coupling in Eq. (23) vanishes, $\mathcal{L}_{\alpha \beta}^{(s t)}=0$. The linearized Boltzmann equation for the total and spin distributions, $n_{\mathbf{p}}$ and $n_{\mathbf{p} s}$, respectively defined by $n_{\sigma}=\left(n+\sigma n_{s}\right) / 2$, also decouples. Defining the corresponding average and spin components as

$$
\Phi_{\mathbf{p} \sigma}=\frac{\phi_{\mathbf{p} t}+\sigma \phi_{\mathbf{p} s}}{2}, \quad \mathcal{C}_{\mathbf{p} \sigma}=\frac{C_{\mathbf{p} t}+\sigma C_{\mathbf{p} s}}{2},
$$

the collision integrals are given by

$$
\begin{aligned}
& \left(\begin{array}{c}
C_{\mathbf{p}_{1} t} \\
C_{\mathbf{p}_{1} s}
\end{array}\right) \\
& =-\int \frac{d \mathbf{p}_{2}}{(2 \pi \hbar)^{3}}\left|\mathbf{v}_{r}\right| f_{1}^{0} f_{2}^{0} \int d \Omega_{r}^{\prime}\left(1+\zeta f_{3}^{0}\right)\left(1+\zeta f_{4}^{0}\right) \\
& \quad \times\left(\begin{array}{c}
\left(d \sigma_{++} / d \Omega_{r}^{\prime}+d \sigma_{+-} / d \Omega_{r}^{\prime}\right) \Delta_{++}\left[\phi_{\mathbf{p} t}\right] \\
\left(d \sigma_{++} / d \Omega_{r}^{\prime}\right) \Delta_{++}\left[\phi_{\mathbf{p} s}\right]+\left(d \sigma_{+-} / d \Omega_{r}^{\prime}\right) \Delta_{+-}\left[\phi_{\mathbf{p} s}\right]
\end{array}\right),
\end{aligned}
$$

where we introduced the notation

$$
\begin{aligned}
& \Delta_{++}\left[\chi_{\mathbf{p}}\right]=\chi_{\mathbf{p}_{3}}+\chi_{\mathbf{p}_{4}}-\chi_{\mathbf{p}_{1}}-\chi_{\mathbf{p}_{2}}, \\
& \Delta_{+-}\left[\chi_{\mathbf{p}}\right]=\chi_{\mathbf{p}_{3}}-\chi_{\mathbf{p}_{4}}-\chi_{\mathbf{p}_{1}}+\chi_{\mathbf{p}_{2}},
\end{aligned}
$$

for a generic momentum-dependent function $\chi_{\mathbf{p}}$. The intraspin term satisfies $\Delta_{++}[\mathbf{p}]=0$ and $\Delta_{++}\left[\epsilon_{\mathbf{p}}\right]=0$, reflecting momentum and energy conservation, while $\Delta_{++}[1]=0$ and $\Delta_{+-}[1]=0$. We note that from Eq. (26), the Boltzmann equation for the total distribution is the same as that of a one-component gas with the interspin and intraspin differential cross sections added together, and the corresponding problem has been studied extensively in the literature [33]. Henceforth, we focus on the spin component.

The spin components of Eqs. (18) and (19) are

$$
\begin{aligned}
\phi_{\mathbf{p} s} & =\boldsymbol{\phi}_{F}(\mathbf{p}) \cdot \mathbf{F}_{s}+\boldsymbol{\phi}_{T}(\mathbf{p}) \cdot\left(-k_{B} \nabla T_{s}\right), \\
C_{\mathbf{p} s}\left[\phi_{s}\right] & =C_{\mathbf{p} s}\left[\boldsymbol{\phi}_{F}\right] \cdot \mathbf{F}_{s}+C_{\mathbf{p} s}\left[\boldsymbol{\phi}_{T}\right] \cdot\left(-k_{B} \nabla T_{s}\right),
\end{aligned}
$$

and the spin component of Eq. (20) is

$$
\mathbf{v}_{\mathbf{p}}=\frac{C_{\mathbf{p} s}\left[\boldsymbol{\phi}_{F}\right]}{\partial_{\epsilon} f_{\mathbf{p}}^{0}}, \quad\left(\frac{\epsilon_{\mathbf{p}}-w}{k_{B} T}\right) \mathbf{v}_{\mathbf{p}}=\frac{C_{\mathbf{p} s}\left[\boldsymbol{\phi}_{T}\right]}{\partial_{\epsilon} f_{\mathbf{p}}^{0}},
$$

where we write $\phi_{\mathbf{p} s} \equiv \sum_{\alpha} \boldsymbol{\phi}_{\alpha} \cdot \mathbf{X}_{\alpha}^{(s)}$, with $\mathbf{X}_{\alpha}^{(s)}$ being the spin component of the thermodynamic forces. Since the collision integral is a linear integral operator, it will be convenient to introduce the notation

$$
\hat{C}_{s} \chi_{\mathbf{p}} \equiv \frac{C_{\mathbf{p} s}[\chi]}{\partial_{\epsilon} f_{\mathbf{p}}^{0}}
$$

for a generic momentum-dependent function $\chi_{\mathbf{p}}$. Then Eq. (28) can be regarded as an eigenvalue equation for the collision integral operator, and solving it amounts to inverting the collision operator $\hat{C}_{s}$. In Sec. V, we will solve Eq. (20) for $\boldsymbol{\phi}_{F}$ and $\boldsymbol{\phi}_{T}$ using a moment expansion.

The spin and spin-heat currents are given by

$$
\begin{aligned}
\left(\begin{array}{l}
\mathbf{j}_{s} \\
\mathbf{q}_{s}
\end{array}\right) & \equiv\left\langle\left(\begin{array}{c}
1 \\
\epsilon_{\mathbf{p}}-w
\end{array}\right) \mathbf{v}_{\mathbf{p}} \phi_{\mathbf{p} s}\right\rangle \\
& \equiv\left(\begin{array}{cc}
\mathcal{L}_{F F}^{(s)} & k_{B} \mathcal{L}_{F T}^{(s)} \\
k_{B} T \mathcal{L}_{T F}^{(s)} & k_{B}^{2} T \mathcal{L}_{T T}^{(s)}
\end{array}\right)\left(\begin{array}{c}
\mathbf{F}_{s} \\
-\nabla T_{s}
\end{array}\right), \\
\langle\phi \chi\rangle & \equiv-\int \frac{d^{3} p}{(2 \pi \hbar)^{3}} \partial_{\epsilon} f_{\mathbf{p}}^{0} \phi_{\mathbf{p}} \chi_{\mathbf{p}} .
\end{aligned}
$$

Henceforth, we drop the superscript $(s)$ for $L_{\alpha \beta}=\mathcal{L}_{\alpha \beta}^{(s)}$. The response coefficients are given in terms of the spin 
distributions by

$$
\begin{aligned}
L_{F \beta} & =\left\langle\mathbf{v}_{\mathbf{p}} \otimes \boldsymbol{\phi}_{\beta}\right\rangle=\left\langle\hat{C}_{s} \boldsymbol{\phi}_{F} \otimes \boldsymbol{\phi}_{\beta}\right\rangle, \\
L_{T \beta} & =\left\langle\frac{\epsilon_{\mathbf{p}}-w}{k_{B} T} \mathbf{v}_{\mathbf{p}} \otimes \boldsymbol{\phi}_{\beta}\right\rangle=\left\langle\hat{C}_{s} \boldsymbol{\phi}_{T} \otimes \boldsymbol{\phi}_{\beta}\right\rangle .
\end{aligned}
$$

In Eq. (31), we expressed $L_{\alpha \beta}$ in terms of the collision integrals using Eq. (28). They are symmetric by the symmetry of the collision integral operator [33],

$$
L_{F T}=\left\langle\hat{C}_{s} \boldsymbol{\phi}_{F} \otimes \boldsymbol{\phi}_{T}\right\rangle=\left\langle\boldsymbol{\phi}_{F} \otimes \hat{C}_{s} \boldsymbol{\phi}_{T}\right\rangle=L_{T F},
$$

and thus satisfy the Onsager reciprocity principle. by

Finally, it is conventional to define the transport coefficients

$$
\left(\begin{array}{c}
\mathbf{j}_{s} \\
\mathbf{q}_{s}
\end{array}\right) \equiv \sigma_{s}\left(\begin{array}{cc}
1 & S_{s} \\
P_{s} & \kappa_{s}^{\prime} / \sigma_{s}
\end{array}\right)\left(\begin{array}{c}
\mathbf{F}_{s} \\
-\nabla T_{s}
\end{array}\right),
$$

which are related to the coefficients $L_{\alpha \beta}$ by

$$
\sigma_{s}=L_{F F}, \quad S_{s}=\frac{P_{s}}{T}=k_{B} \frac{L_{F T}}{L_{F F}}, \quad \kappa_{s}^{\prime}=k_{B}^{2} T L_{T T} .
$$

Furthermore, we define the spin-heat conductivity at zero spin current $\kappa_{s}$ and a figure of merit for thermospin conversion $Z_{\mathrm{s}} T$, given by [36]

$$
\begin{aligned}
\kappa_{s} & =\kappa_{s}^{\prime}-\sigma_{s} S_{s}^{2} T=k_{B}^{2} T \frac{\operatorname{det} \hat{L}}{L_{F F}}, \\
Z_{s} T & =\frac{\sigma_{s} S_{s}^{2} T}{\kappa_{s}}=\frac{L_{F T}^{2}}{\operatorname{det} \hat{L}},
\end{aligned}
$$

where $\hat{L}$ is the matrix of response coefficients $L_{\alpha \beta}$. The response coefficients in (32) can be accessed directly in experiments. The spin-Seebeck effect, for example, can be measured in the manner discussed in Ref. [37].

\section{Relaxation coefficients}

We now derive a microscopic expression for the spin-heat relaxation rate $1 / \tau_{\mathrm{st}}$ and length $\lambda_{\mathrm{st}}$. As mentioned previously, this relaxation term comes from the energy transfer between spins represented by $\Gamma_{\sigma}\left[\delta \vec{f}_{\mathbf{p}}\right]$ in Eq. (8), where $\delta \vec{f}_{\mathbf{p}}$ is the perturbation to the local equilibrium distribution due to the spin-heat accumulation $T_{s}$, given to leading order by

$$
\delta f_{\mathbf{p} \sigma}(t)=\frac{\sigma T_{s}}{2} \partial_{\epsilon} f_{\mathbf{p} \sigma}^{0} \frac{\epsilon_{\mathbf{p}}-w}{k_{B} T} .
$$

Recalling that the energy is a collisional invariant [see Eq. (9)], in $\mathcal{C}_{\mathbf{p} \sigma}\left[\delta f_{\mathbf{p} \sigma}(t)\right]$, only the spin component $C_{\mathbf{p} s}$ is nonzero; thus,

$$
\Gamma_{\sigma}\left[\delta \vec{f}_{\mathbf{p}}\right]=\frac{\sigma T_{s}}{2}\left\langle\epsilon_{\mathbf{p}} \hat{C}_{s}\left(\epsilon_{\mathbf{p}} / k_{B} T\right)\right\rangle .
$$

Thus, the spin-heat relaxation rate and length [see Eq. (13)] are given by

$$
\begin{aligned}
\frac{1}{\tau_{\mathrm{st}}} & =\frac{\left\langle\epsilon_{\mathbf{p}} \hat{C}_{s}\left(\epsilon_{\mathbf{p}} / k_{B} T\right)\right\rangle}{\rho c_{p}} \\
\lambda_{\mathrm{st}} & =\sqrt{\frac{\kappa_{s}^{\prime} \tau_{\mathrm{st}}}{\rho c_{p}}}=\sqrt{\frac{k_{B}^{2} T L_{T T}}{\left\langle\epsilon_{\mathbf{p}} \hat{C}_{s}\left(\epsilon_{\mathbf{p}} / k_{B} T\right)\right\rangle}} .
\end{aligned}
$$

\section{Total entropy production}

In this section, we compute the total entropy production due to spin-heat accumulation gradients and spin forces. It can be conveniently computed directly from the nonequilibrium entropy density defined in terms of the distribution function, given by

$$
\begin{aligned}
\rho_{\sigma} s_{\sigma}= & k_{B} \int \frac{d \mathbf{p}}{(2 \pi \hbar)^{3}}\left[\zeta\left(1+\zeta n_{\mathbf{p} \sigma}\right) \ln \left(1+\zeta n_{\mathbf{p} \sigma}\right)\right. \\
& \left.-n_{\mathbf{p} \sigma} \ln n_{\mathbf{p} \sigma}\right] .
\end{aligned}
$$

The entropy density production is thus

$$
\partial_{t}\left(\rho_{\sigma} s_{\sigma}\right)=k_{B} \int \frac{d \mathbf{p}}{(2 \pi \hbar)^{3}} \partial_{t} n_{\mathbf{p} \sigma} \ln \left(\frac{1+\zeta n_{\mathbf{p} \sigma}}{n_{\mathbf{p} \sigma}}\right) .
$$

The total entropy production is the equation above integrated over all space. It contains contributions from only the collision integral. Using the Boltzmann equation (1) and the ansatz (14), we find the heating given by the quadratic form

$$
\begin{aligned}
T & \sum_{\sigma} \int d \mathbf{r} \partial_{t}\left(\rho_{\sigma} s_{\sigma}\right)_{\mathrm{coll}} \\
& =\frac{1}{2} \int d \mathbf{r}\left\langle\phi_{\mathbf{p} s} \hat{C}_{s} \phi_{\mathbf{p} s}\right\rangle \\
& =\frac{1}{2} \int d \mathbf{r} \sum_{\alpha, \beta} \mathbf{X}_{\alpha} \cdot L_{\alpha \beta} \cdot \mathbf{X}_{\beta} \\
& =\int d \mathbf{r}\left(\frac{L_{F F}}{2} \mathbf{F}_{s}^{2}+\frac{k_{B}^{2} L_{T T}}{2} \nabla T_{s}^{2}+k_{B} L_{F T} \mathbf{F}_{s} \cdot \nabla T_{s}\right)
\end{aligned}
$$

Measuring this heating will provide an indirect measurement of the response coefficients $L_{F F}, L_{F T}$, and $L_{T T}$.

\section{SOLUTION BY MOMENT EXPANSION}

To solve the steady-state Boltzmann equation (28), we use a polynomial expansion [38],

$$
\boldsymbol{\phi}_{\alpha}=\sum_{n=0} c_{n}^{(\alpha)}\left(\frac{\epsilon_{\mathbf{p}}}{k_{B} T}\right)^{n} \mathbf{p}, \quad \alpha=F, T .
$$

Taking the $m$ th moment by

$$
-\int \frac{d^{3} p}{(2 \pi \hbar)^{3}}\left(\frac{\epsilon_{\mathbf{p}}}{k_{B} T}\right)^{n} \mathbf{p} \cdot \text { Eq. (28), }
$$

with $m=0,1,2, \ldots$, results in a set of equations

$$
\frac{3}{\Lambda^{3}} l_{m}^{(\alpha)}=\sum_{n=0}^{\infty} \mathrm{C}_{m n} c_{n}^{(\alpha)}
$$

where the matrix elements of the collision operator are

$$
\begin{aligned}
\mathrm{C}_{m n} & =\left\langle\left(\frac{\epsilon_{\mathbf{p}}}{k_{B} T}\right)^{m} \mathbf{p} \cdot \hat{C}_{s}\left(\frac{\epsilon_{\mathbf{p}}}{k_{B} T}\right)^{n} \mathbf{p}\right\rangle \\
& =-\int \frac{d^{3} p}{(2 \pi \hbar)^{3}}\left(\frac{\epsilon_{\mathbf{p}}}{k_{B} T}\right)^{m} \mathbf{p} \cdot C_{\mathbf{p} s}\left[\left(\frac{\epsilon_{\mathbf{p}}}{k_{B} T}\right)^{n} \mathbf{p}\right],
\end{aligned}
$$


where a dot (.) denotes a dot product and we defined the following functions:

$$
\begin{aligned}
l_{m}^{(F)} & =l_{m}, \quad l_{m}^{(T)}=l_{m+1}-\frac{w}{k_{B} T} l_{m}, \\
l_{n} & \equiv \Lambda^{3}\left\langle\mathbf{p} \otimes \mathbf{v}_{p}\left(\frac{\epsilon_{\mathbf{p}}}{k_{B} T}\right)^{n}\right\rangle=\zeta \frac{\Gamma_{n+5 / 2}}{\Gamma_{5 / 2}} \operatorname{Li}_{n+3 / 2}(\zeta z),
\end{aligned}
$$

where $\Lambda=\hbar \sqrt{2 \pi / m k_{B} T}$ is the de Broglie wavelength, $z=$ $e^{\mu} / T$ is the fugacity, $\operatorname{Li}_{s}(z)=\sum_{n=1}^{\infty} z^{n} / n^{s}$ are the polylogarithmic functions, and $\Gamma_{n}$ denotes the gamma function. The first two $l_{n}$ 's can be expressed in terms of thermodynamic quantities (see Appendix A),

$$
\left(\begin{array}{l}
l_{0} \\
l_{1}
\end{array}\right)=\zeta\left(\begin{array}{c}
\mathrm{Li}_{3 / 2}(\zeta \mathrm{z}) \\
\frac{5}{2} \mathrm{Li}_{5 / 2}(\zeta z)
\end{array}\right)=\rho \Lambda^{3}\left(\begin{array}{c}
1 \\
w / k_{B} T
\end{array}\right) .
$$

The expansion coefficients that follow from inverting Eq. (38) are

$$
c_{n}^{(\alpha)}(z, \Lambda)=\frac{3}{\Lambda^{3}} \sum_{m} \mathrm{C}_{n m}^{-1}(z, \Lambda) l_{m}^{(\alpha)}(z) .
$$

The response coefficients follow from substituting the expansion (37) in Eq. (31) [39],

$$
L_{\alpha \beta}=\frac{1}{\Lambda^{3}} \sum_{n} c_{n}^{(\beta)} l_{n}^{(\alpha)} .
$$

Truncating this expansion at the second order [40], the $L_{\alpha \beta}$ coefficients are

$$
\begin{aligned}
L_{F F} & =\rho\left(c_{0}^{(F)}+c_{1}^{(F)} \frac{w}{k_{B} T}\right), \\
L_{F T} & =\rho\left(c_{0}^{(T)}+c_{1}^{(T)} \frac{w}{k_{B} T}\right), \\
L_{T F} & =\rho c_{1}^{(F)} f(z, T), \\
L_{T T} & =\rho c_{1}^{(T)} f(z, T), \\
f(z, T) & \equiv \frac{l_{1}^{(T)}}{\rho \Lambda^{3}}=\left[\frac{35}{4} \frac{\mathrm{Li}_{7 / 2}(\zeta z)}{\operatorname{Li}_{3 / 2}(\zeta z)}-\left(\frac{w}{k_{B} T}\right)^{2}\right] .
\end{aligned}
$$

Note that since $l_{0}^{(T)}=0$, the heat current, proportional to $L_{T T}$ and $L_{T F}$, does not depend on $c_{0}^{(F)}$ and $c_{0}^{(T)}$. A comparison of this solution with the one used to compute the spin-drag relaxation time in the literature in the absence of spin-heat currents is given in Appendix C [28,29].

We conclude this section by verifying that our approximate solution satisfies Onsager reciprocity. Using Eq. (42) to express the transport coefficients in terms of the collision matrix elements,

$$
\begin{aligned}
L_{F F}= & \frac{3}{\Lambda^{6}} \sum_{m n} \mathrm{C}_{n m}^{-1} l_{n} l_{m}, \\
L_{F T}= & \frac{3}{\Lambda^{6}} \sum_{m n} \mathrm{C}_{n m}^{-1} l_{n} l_{m+1}-\frac{w}{k_{B} T} L_{F F}, \\
L_{T F}= & \frac{3}{\Lambda^{6}} \sum_{m n} \mathrm{C}_{n m}^{-1} l_{n+1} l_{m}-\frac{w}{k_{B} T} L_{F F}, \\
L_{T T}= & \frac{3}{\Lambda^{6}} \sum_{n m} \mathrm{C}_{n m}^{-1}\left(l_{n+1} l_{m+1}-2 \frac{w}{k_{B} T} l_{n} l_{m+1}\right) \\
& +\left(\frac{w}{k_{B} T}\right)^{2} L_{F F} .
\end{aligned}
$$

Since $\mathrm{C}_{n m}$ is symmetric, so is $\mathrm{C}_{n m}^{-1}$, and thus, we satisfy the Onsager relation, $L_{F T}=L_{T F}$, order by order in this expansion.

\section{TRANSPORT AND RELAXATION COEFFICIENTS FOR $s$-WAVE SCATTERING}

In this section, we present our results for spin-heat transport coefficients as a function of temperature and interaction strength for the $s$-wave scattering differential cross section. We numerically evaluate the second-order formulas for the response coefficients given in Eq. (44). The computation of the required collision matrix elements $\mathrm{C}_{n m}$ is detailed in Appendix B.

To gain some analytical insight into our numerical results, we use scaling arguments to deduce the form of transport coefficients as a function of temperature and scattering length. We first factor out the generic temperature dependence by expressing the collision matrix elements as a function of the dimensionless momentum $\tilde{\mathbf{p}}=(\Lambda / \sqrt{4 \pi} \hbar) \mathbf{p}$ and define a dimensionless $s$-wave scattering cross section by

$$
\frac{d \tilde{\sigma}_{+-}}{d \Omega}=\Lambda^{-2} \frac{d \sigma_{+-}}{d \Omega}=\frac{(a / \Lambda)^{2}}{1+4 \pi(a / \Lambda)^{2} \tilde{p}_{r}^{2}},
$$

where, here and below, we denote dimensionless quantities by a tilde. With this rescaling, we define dimensionless collision matrix elements by

$$
\mathrm{C}_{n m} \equiv \frac{\hbar}{\Lambda^{5}} \tilde{\mathrm{C}}_{n m}\left(\frac{\lambda_{\zeta}}{\Lambda}, \frac{a}{\Lambda}\right)
$$

Here, we express the fugacity $z=\zeta \mathrm{Li}_{3 / 2}^{-1}\left(\zeta \rho \Lambda^{3}\right)$ as a function of $\lambda_{\zeta}$, where we define $\lambda_{1}=\lambda_{B}=\rho^{-1 / 3}$ for bosons, $\lambda_{-1}=$ $\lambda_{F}=2 \pi\left(6 \pi^{2} \rho\right)^{-1 / 3}$ is the Fermi wavelength, and $\rho$ is the equilibrium density. Equation (B4) gives the explicit expression for $\tilde{\mathrm{C}}_{n m}$. Then, from Eq. (45), the transport coefficients have the scaling form

$$
\hbar \lambda_{\zeta} L_{\alpha \beta} \equiv \frac{\lambda_{\zeta}}{\Lambda} \tilde{L}_{\alpha \beta}\left(\frac{\lambda_{\zeta}}{\Lambda}, \frac{a}{\Lambda}\right)
$$

where $\tilde{L}_{\alpha \beta} \propto \tilde{\mathrm{C}}_{n m}^{-1} l_{m} l_{n}$. Then, we express the parameters above as functions of temperature as

$$
\frac{\lambda_{\zeta}}{\Lambda}=y_{\zeta}^{-1 / 3} \sqrt{\frac{T}{T_{\zeta}}}, \quad \frac{a}{\Lambda}=\frac{a}{\lambda_{\zeta}} y_{\zeta}^{-1 / 3} \sqrt{\frac{T}{T_{\zeta}}},
$$

where $T_{1}=T_{c}$ is the critical temperature for Bose-Einstein condensation given by $\rho \Lambda_{c}^{3}=\mathrm{Li}_{3 / 2}(1) \simeq 2.612$, while for fermions, $T_{-1}=T_{F}$ is the Fermi temperature defined by $k_{B} T_{F}=(2 \pi \hbar)^{2} / 2 m \lambda_{F}^{2}$. It will also be useful to note the relation $\rho \Lambda^{3}=x_{\zeta}\left(T / T_{\zeta}\right)^{-3 / 2}$, where we define the constants $x_{-1}=4 / 3 \sqrt{\pi}, y_{-1}=\pi^{-3 / 2}$, and $y_{1}=x_{1}=2.612$.

We first define the dimensionless transport coefficients given in terms of dimensionless variables,

$$
\begin{aligned}
& \hbar \lambda_{\zeta} \sigma_{s}=y_{\zeta}{ }^{-1 / 3}\left(\frac{T}{T_{\zeta}}\right)^{1 / 2} \tilde{L}_{F F}, \\
& \frac{\hbar \lambda_{\zeta} \kappa_{s}}{k_{B}^{2} T_{\zeta}}=y_{\zeta}{ }^{-1 / 3}\left(\frac{T}{T_{\zeta}}\right)^{3 / 2} \frac{\operatorname{det} \tilde{L}}{\tilde{L}_{F F}},
\end{aligned}
$$



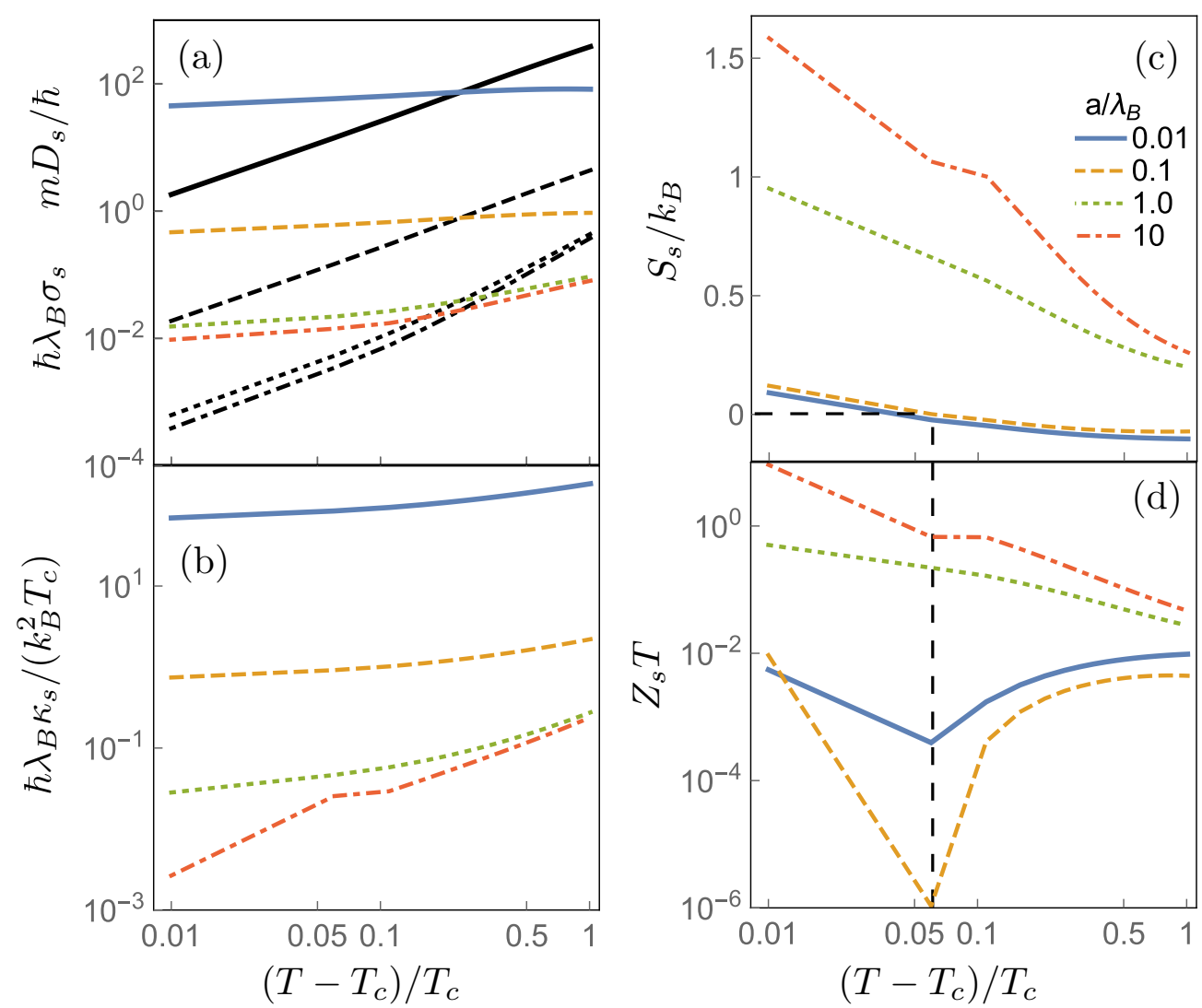

FIG. 3. (Color online) Bosonic spin-heat transport coefficients for the ratios of interspin scattering lengths to interparticle spacing $a / \lambda_{B}=$ $(0.01,0.1,1,10)$. (a) In color (gray): $\log$-log plot of the normalized spin conductivity $\hbar \lambda_{B} \sigma_{s}$. In black: $\log$-log plot of the normalized spin diffusivity $\hbar D_{s} / m$. (b) Log-log plot of the normalized spin-heat conductivity $\hbar \lambda_{B} \kappa_{s} / k_{B}^{2} T_{c}$. (c) Log-linear plot of the spin-Seebeck coefficient in units of the Boltzmann constant, $S_{s} / k_{B}$. Dashed black line indicates the zero crossing for weak-scattering lengths $a / \lambda_{B}=(0.01,0.1)$. (d) Log-log plot of the spin-heat figure of merit $Z_{s} T$.

$$
\begin{aligned}
\frac{S_{s}}{k_{B}} & =\frac{\tilde{L}_{F T}}{\tilde{L}_{F F}}, \\
Z_{S} T & =\frac{\tilde{L}_{F T}^{2}}{\operatorname{det} \tilde{L}} .
\end{aligned}
$$

We plot these coefficients in Fig. 3 for bosons and refer the reader to Ref. [37] for the corresponding plots for fermions. Figure 3(a) shows the spin conductivity [in color (gray)] together with the spin diffusivities (in black); the spin diffusivity is the transport coefficient measured in experiments as it determines the spin current driven by spin-density gradients via $\mathbf{j}_{s}=-D_{s} \nabla \rho_{s}$ [10]. It is related to the spin conductivity by $D_{s}=\sigma_{s} / \chi_{s}$, where $\chi_{s}=\partial \rho_{s} / \partial \mu_{s}$ is the static spin susceptibility and $\mu_{s}=\mu_{+}-\mu_{-}$is the spin accumulation; expressed in units of $\hbar / m$, it is given by

$$
\frac{m}{\hbar} D_{s}=\frac{m}{\hbar} \frac{\sigma_{s}}{\chi_{s}}=\frac{2 \pi \tilde{\sigma}_{s}}{\zeta \operatorname{Li}_{\frac{1}{2}}(\zeta z)},
$$

where $\tilde{\sigma}_{s}=\hbar \Lambda \sigma_{s}$. The decrease in $\sigma_{s}$ and $D_{s}$ as a function of $T-T_{c}$ and $a$ is due to the Bose enhancement of scattering. The spin-heat conductivity at zero current $\kappa_{s}$, plotted in Fig. 3(b), behaves similarly.

The spin-Seebeck coefficient and thermospin figure of merit $Z_{S} T$ are plotted in Figs. 3(c) and 3(d). For strong scattering $\left(a / \lambda_{B} \geqslant 1\right)$, these coefficients are strongly enhanced near the critical temperature but at the cost of much shorter spin-heat relaxation lengths $\lambda_{\mathrm{st}}<1 \mu \mathrm{m}$. For weak scattering $\left(a / \lambda_{B} \leqslant 1\right)$, we find $S_{s} / k_{B} \simeq 0.1$ at $\left(T-T_{c}\right) / T_{c}=0.01$, which is much larger than the case for fermions [37]. We also note, for weak scattering, a sign change occurs in the spin-Seebeck coefficient as a function of temperature [37]. In contrast, for fermions the spin-Seebeck coefficient changes sign for strong scattering $\left(a / \lambda_{F} \geqslant 1\right)$. We attribute the sign change to a crossover from particle- to hole-dominated transport, as discussed in Ref. [37].

For bosons in the degenerate limit, according to the dynamical theory of critical phenomena [41], transport coefficients exhibit power-law behavior. This can be seen by rescaling lengths by the correlation length $\xi$,

$$
\hbar L_{\alpha \beta} \equiv \frac{1}{\xi} \tilde{L}_{\alpha \beta}\left(\frac{\lambda}{\xi}, \frac{\Lambda}{\xi}, \frac{a}{\xi}\right),
$$

where [42] [see Eq. (A5)]

$$
\xi=\frac{\Lambda}{2 \pi^{1 / 2}}\left(\frac{-\mu}{k_{B} T}\right)^{-1 / 2} .
$$

Defining $t=\left(T-T_{c}\right) / T_{c}$, the correlation length diverges as $\xi \sim t^{-1}$ [see Eq. (A7)]. Since $\tilde{L}_{\alpha \beta}$ is analytic in $\lambda, a$, at degenerate temperatures, a power-law dependence on $\xi$ 


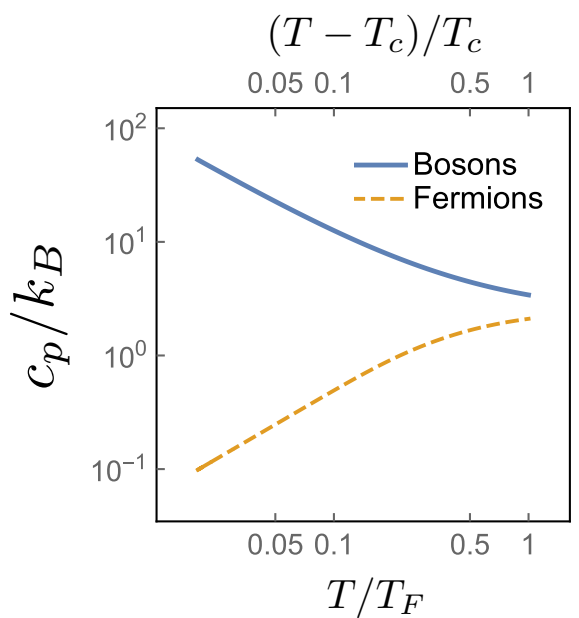

FIG. 4. (Color online) The specific heat capacity at constant pressure in units of $k_{B}, c_{p} / k_{B}$, of the ideal Bose and Fermi gases as a function of $\left(T-T_{c}\right) / T_{c}$ and $T / T_{F}$, respectively.

and $t$ follows from the scaling relation (47). The critical phenomenon associated with the two-component gas at equal density was studied in Ref. [41], where it was called "the symmetric binary fluid."

In the high-temperature limit, the transport coefficients have the scaling form

$$
\hbar L_{\alpha \beta} \equiv \frac{1}{\Lambda} \tilde{L}_{\alpha \beta}\left(\frac{a}{\Lambda}\right),
$$

which is the same for bosons and fermions. As shown in Eq. (A4), $\Lambda$ is the correlation length in this limit. We refer to Fig. 4 of Ref. [37] for plots of the transport coefficients in the high-temperature limit as functions of $a / \Lambda$.

The spin-heat relaxation rate and length [see Eq. (35)], in units of $k_{B} T_{\zeta} / \hbar$ and in units of $\lambda_{\zeta}$, respectively, are given in terms of dimensionless variables by

$$
\begin{gathered}
\frac{\hbar}{k_{B} T_{\zeta} \tau_{\mathrm{st}}}=\frac{1}{x_{\zeta}}\left(\frac{T}{T_{\zeta}}\right)^{5 / 2} \frac{\tilde{\mathrm{C}}_{\mathrm{st}}(z, a / \Lambda)}{c_{p}(\zeta z)}, \\
\frac{\lambda_{\mathrm{st}}}{\lambda_{\zeta}}=\frac{1}{\lambda_{\zeta}} \sqrt{\frac{\kappa_{s}^{\prime} \tau_{\mathrm{st}}}{\rho c_{p}}}=y_{\zeta}^{1 / 3} \sqrt{\frac{T_{\zeta}}{T}} \sqrt{\frac{\tilde{L}_{T T}}{\tilde{\mathrm{C}}_{\mathrm{st}}}},
\end{gathered}
$$

where we defined

$$
\tilde{\mathrm{C}}_{\mathrm{st}}(z, a / \Lambda) \equiv \Lambda^{3} \hbar\left\langle\left(\frac{\epsilon}{k_{B} T}\right) \hat{C}_{s}\left(\frac{\epsilon}{k_{B} T}\right)\right\rangle
$$

and the heat capacity at constant pressure $c_{p}$ is given in Eq. (A2) and is plotted in Fig. 4. These relaxation coefficients are plotted in Fig. 2, and their qualitative behavior is discussed in Sec. III. As mentioned before, for bosons the divergence in $\tau_{\text {st }}$ stems from the divergence of the heat capacity, plotted in Fig. 4 , as $T \rightarrow T_{c}$. For fermions, where the heat capacity remains finite [see Fig. 4(b)], the downturn occurs because of Pauli blocking, which inhibits scattering. Thus, we find that the relaxation times diverge at degenerate temperatures, so that the spin-heat accumulation is, in principle, well defined for degenerate gases.

\section{CONCLUSION AND OUTLOOK}

In summary, we have developed the theory of coupled spin and heat transport in ultracold atomic gases at degenerate temperatures, including quantum effects due to Bose and Fermi statistics and quantum-mechanical scattering. Using a perturbative solution to the Boltzmann equations that explicitly respects the Onsager reciprocity principle, we computed the spin-heat transport and relaxation coefficients. We find a divergence in the spin-heat relaxation times at degenerate temperatures, and we find that the spin-heat relaxation lengths can be of the order of millimeters. This raises the hope that the spin-to-heat conversion studied in this work and Refs. [20,37] can be achieved in ultracold atom experiments. Specifically, using the spin-Seebeck coupling, pure spin-heat currents and resulting spin-dependent heating can be generated by spin forces even in a gas with equal densities of spin-up and spin-down particles.

In this work, we have only touched upon the spin hydrodynamics of two-component gases, which is rich and complex even in the classical regime, and experimental efforts in this direction have only recently begun. We expect much more interesting and possibly useful physics to emerge in relation to this subject, and we hope this work will motivate further experimental efforts to study thermospin effects in ultracold atomic gases.

\section{ACKNOWLEDGMENTS}

This work was supported by the Stichting voor Fundamenteel Onderzoek der Materie (FOM) and by the European Research Council (ERC) under the Seventh Framework Program (FP7) and is part of the D-ITP Consortium, a program of the Netherlands Organisation for Scientific Research (NWO) that is funded by the Dutch Ministry of Education, Culture and Science (OCW).

\section{APPENDIX A: THERMODYNAMIC PROPERTIES}

In this Appendix, we summarize the equilibrium properties of noninteracting degenerate gases. The local density [43], energy density, pressure, and entropy per particle are given by

$$
\begin{aligned}
\left(\begin{array}{c}
\rho_{\sigma}\left(\mu_{\sigma}, T_{\sigma}\right) \\
e_{\sigma}\left(\mu_{\sigma}, T_{\sigma}\right)
\end{array}\right) & =\int \frac{d^{3} p}{(2 \pi \hbar)^{3}}\left(\begin{array}{c}
1 \\
\epsilon_{\mathbf{p}}
\end{array}\right) \frac{1}{z_{\sigma}^{-1} e^{\epsilon_{\mathbf{p}} / k_{B} T_{\sigma}}-\zeta} \\
& =\frac{\zeta}{\Lambda^{3}}\left(\begin{array}{c}
\mathrm{Li}_{3 / 2}\left(\zeta \mathrm{z}_{\sigma}\right) \\
\left(3 k_{B} T / 2\right) \mathrm{Li}_{5 / 2}(\zeta z)
\end{array}\right), \\
p_{\sigma}\left(\mu_{\sigma}, T_{\sigma}\right) & =k_{B} \int \frac{d^{3} p}{(2 \pi)^{3}} \ln \left(1-e^{\left(\epsilon_{\mathbf{p}}-\mu_{\sigma}\right) / k_{B} T_{\sigma}}\right) \\
& =\operatorname{Li}_{5 / 2}(z) \frac{k_{B} T}{\Lambda^{3}}, \\
\frac{s_{\zeta}(z)}{k_{B}} & =\frac{5}{2} \frac{\operatorname{Li}_{5 / 2}(\zeta z)}{\operatorname{Li}_{3 / 2}(\zeta z)}-\ln z,
\end{aligned}
$$

where $\Lambda=\hbar \sqrt{2 \pi / m k_{B} T}$ is the thermal de Broglie wavelength and $z_{\sigma}=e^{\mu_{\sigma} / k_{B} T_{\sigma}}$ is the fugacity. The pressure is related to the energy density by $p_{\sigma}=2 e_{\sigma} / 3$, giving the equation of 
state

$$
p_{\sigma}\left(\rho_{\sigma}, T_{\sigma}\right)=\rho_{\sigma} k_{B} T_{\sigma} \frac{\mathrm{Li}_{5 / 2}\left(\zeta z_{\sigma}\right)}{\mathrm{Li}_{3 / 2}\left(\zeta z_{\sigma}\right)},
$$

where in the above equation the chemical potential is meant to be expressed in terms of the density by

$$
\mu_{\sigma}\left(\rho_{\sigma}, T_{\sigma}\right)=\zeta k_{B} T_{\sigma} \mathrm{Li}_{3 / 2}^{-1}\left(\zeta \rho_{\sigma} \Lambda^{3}\right) .
$$

The energy and enthalpy per particle, $u_{\sigma} \equiv e_{\sigma} / \rho_{\sigma}$ and $w_{\sigma} \equiv$ $u_{\sigma}+p_{\sigma} / \rho_{\sigma}=\frac{5}{3} u_{\sigma}$, respectively, are given by

$$
\left(\begin{array}{l}
u_{\sigma}\left(\mu_{\sigma}, T_{\sigma}\right) \\
w_{\sigma}\left(\mu_{\sigma}, T_{\sigma}\right)
\end{array}\right)=\left(\begin{array}{l}
3 / 2 \\
5 / 2
\end{array}\right) k_{B} T_{\sigma} \frac{\mathrm{Li}_{5 / 2}\left(\zeta z_{\sigma}\right)}{\operatorname{Li}_{3 / 2}\left(\zeta z_{\sigma}\right)} .
$$

The polylogarithms arise through the integrals

$$
\int_{0}^{\infty} d x \frac{x^{s-1}}{z^{-1} e^{x}-\zeta}=\zeta \Gamma_{s} \operatorname{Li}_{s}(\zeta z),
$$

where

$$
\Gamma_{s}=\int_{0}^{\infty} d x x^{s-1} e^{-x}
$$

is the gamma function. The polylogarithms have the series expansion

$$
\operatorname{Li}_{s}(z)=\sum_{n=1}^{\infty} \frac{z^{n}}{n^{s}}=z+\frac{z^{2}}{2^{s}}+\cdots
$$

and satisfy the recursion relations

$$
z \partial_{z} \mathrm{Li}_{s}=\mathrm{Li}_{s-1}
$$

The specific heat capacity at constant pressure can be expressed in terms of the enthalpy change as

$$
c_{p}=T\left(\frac{\partial s}{\partial T}\right)_{p}=\left(\frac{\partial w}{\partial T}\right)_{p} .
$$

Using the formulas in Eq. (A1) and the identity $(\partial \mu / \partial T)_{p}=-s$, we find [42]

$$
\frac{c_{p}(\zeta z)}{k_{B}}=\frac{25}{4} \frac{\mathrm{Li}_{\frac{1}{2}}(\zeta z) \mathrm{Li}_{\frac{5}{2}}^{2}(\zeta z)}{\operatorname{Li}_{\frac{3}{2}}^{3}(\zeta z)}-\frac{15}{4} \frac{\mathrm{Li}_{\frac{5}{2}}(\zeta z)}{\mathrm{Li}_{\frac{3}{2}}(\zeta z)} .
$$

The heat capacities for bosons and fermions are plotted in Figs. 4(a) and 4(b), respectively. For bosons, $c_{p}$ diverges as one approaches the Bose-Einstein phase transition.

We next derive the correlation length in the Bose gas and the classical gas from the local equilibrium distribution for the one-component gas [44]. The one-particle correlation function is related to the semiclassical distribution function by [45]

$$
G(\mathbf{r})=\int \frac{d \mathbf{p}}{(2 \pi \hbar)^{3}} \frac{e^{i \mathbf{p} \cdot \mathbf{r} / \hbar}}{z^{-1} e^{\epsilon_{\mathbf{p}} / k_{B} T}-\zeta} .
$$

In the high-temperature limit $z \ll 1$, one finds

$$
G(r)=\frac{z}{\Lambda^{3}} e^{-\pi r^{2} / \Lambda^{2}},
$$

where $r=|\mathbf{r}|$ so that $\Lambda$ is the correlation length for classical thermal fluctuations.

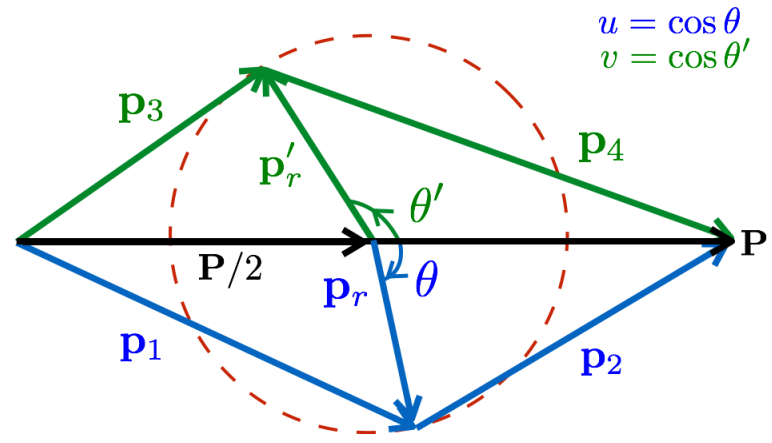

FIG. 5. (Color online) Coordinates for the two-body scattering angles. The red dashed circle indicates the constraint $\left|\mathbf{p}_{r}\right|=\left|\mathbf{p}_{r}\right|^{\prime}$ due to energy conservation.

In the limit of degenerate temperatures, as $T \rightarrow T_{c}$, for $r \ll \xi$, one finds [42]

$$
G(r) \approx \frac{e^{-r / \xi}}{\Lambda^{2}}, \quad \xi=\frac{\Lambda}{2 \pi^{1 / 2}}\left(\frac{-\mu}{k_{B} T}\right)^{-1 / 2}
$$

thus, $\xi$ is the correlation length. To show that it diverges as $T \rightarrow T_{c}^{+}$, consider the asymptotic expansion in the limit $T \rightarrow T_{c}^{+}$,

$$
\operatorname{Li}_{d / 2}(z) \sim \zeta_{d / 2}-\left|\Gamma_{\frac{2-d}{2}}\right|\left(\frac{-\mu}{k_{B} T}\right)^{\frac{d-2}{2}},
$$

where $\zeta_{d / 2}$ is the Riemann zeta function, i.e., $\zeta_{3 / 2}=2.612$, $\zeta_{5 / 2}=1.314$. Then, solving for $\mu$ from $\rho \Lambda^{3}=\mathrm{Li}_{3 / 2}(z)$, one finds

$$
\frac{-\mu}{k_{B} T} \rightarrow\left(\frac{(3 / 2) \zeta_{3 / 2}}{\Gamma_{1 / 2}}\right)^{2} t^{2}, \quad t \equiv \frac{T-T_{c}}{T_{c}}
$$

where $t$ is the reduced temperature. From Eqs. (A7) and (A5), we have $\xi \sim t^{-1}$. Substituting Eq. (A7) into (A2) gives the critical exponent for the power-law dependence of $c_{p}$ on $t$. A similar procedure can be performed to extract the critical exponent for all the bosonic transport coefficients calculated in this paper.

\section{APPENDIX B: EVALUATION OF COLLISION MATRIX ELEMENTS}

To evaluate the collision matrix elements, in Eq. (39), we go to center-of-mass coordinates (see Fig. 5)

$$
\mathbf{p}_{1,2}=\frac{\mathbf{P}}{2} \pm \mathbf{p}_{r}, \quad \mathbf{p}_{3,4}=\frac{\mathbf{P}^{\prime}}{2} \pm \mathbf{p}_{r}^{\prime}
$$

Energy and momentum conservation gives $p_{r} \equiv\left|\mathbf{p}_{r}\right|=\left|\mathbf{p}_{r}^{\prime}\right|$ and $\mathbf{P}=\mathbf{P}^{\prime}$. Furthermore, we use the Hermitian property of the collision operator [33] to express collision matrix elements 
in a symmetric form,

$$
\mathrm{C}_{m n}=\frac{1}{2 m k_{B} T} \int \frac{d \mathbf{P} d \mathbf{p}_{r}}{(2 \pi \hbar)^{6}} p_{r} \int d \Omega_{r} d \Omega_{r}^{\prime} F\left(z ; p_{r}, P, \hat{\mathbf{P}} \cdot \hat{\mathbf{p}_{\mathbf{r}}}, \hat{\mathbf{P}} \cdot \hat{\mathbf{p}_{\mathbf{r}}}\right) \sum_{\sigma}\left\{\frac{d \sigma_{+\sigma}}{d \Omega} \Delta_{+\sigma}\left[\left(\frac{\epsilon_{\mathbf{p}}}{k_{B} T}\right)^{n} \mathbf{p}\right] \cdot \Delta_{+\sigma}\left[\left(\frac{\epsilon_{\mathbf{p}}}{k_{B} T}\right)^{m} \mathbf{p}\right]\right\},
$$

where we define a function of the phase-space occupation functions with energy and momentum conservation enforced as

$$
\begin{aligned}
F\left(z ; p_{r}, P, \hat{\mathbf{P}} \cdot \hat{\mathbf{p}}, \hat{\mathbf{P}} \cdot \hat{\mathbf{p}}_{\mathbf{r}}{ }^{\prime}\right) & \equiv f_{1}^{0} f_{2}^{0}\left(1+\zeta f_{3}^{0}\right)\left(1+\zeta f_{4}^{0}\right) \\
& =\frac{z^{2} e^{\left(\epsilon_{3}+\epsilon_{4}\right) / k_{B} T}}{\left(e^{\epsilon_{1} / k_{B} T}-\zeta z\right)\left(e^{\epsilon_{2} / k_{B} T}-\zeta z\right)\left(e^{\epsilon_{3} / k_{B} T}-\zeta z\right)\left(e^{\epsilon_{4} / k_{B} T}-\zeta z\right)}, \\
\frac{\epsilon_{1,2}}{k_{B} T} & =\frac{P^{2} / 4+p_{r}^{2} \pm P p_{r} \hat{\mathbf{P}} \cdot \hat{\mathbf{p}_{\mathbf{r}}}}{2 m k_{B} T}, \quad \frac{\epsilon_{3,4}}{k_{B} T}=\frac{P^{2} / 4+p_{r}^{2} \pm P p_{r} \hat{\mathbf{P}} \cdot \hat{\mathbf{p}_{\mathbf{r}}}}{2 m k_{B} T},
\end{aligned}
$$

where the fugacity $z=e^{\mu / k_{B} T}$ is determined by the density and temperature. To factor out the dimensionful quantities, here and below, we define dimensionless momenta by the rescaling,

$$
\mathbf{p} \rightarrow \sqrt{2 m k_{B} T} \mathbf{p}=\frac{\sqrt{4 \pi} \hbar}{\Lambda} \mathbf{p} .
$$

Letting $(\theta, \varphi)$ and $\left(\theta^{\prime}, \varphi^{\prime}\right)$ be the spherical angles of $\mathbf{p}_{r}$ and $\mathbf{p}_{r}^{\prime}$, taking the $z$ axis to lie along $\mathbf{P}$ (see Fig. 5), and defining $u=\cos \theta$, $v=\cos \theta^{\prime}$, we have

$$
\begin{gathered}
\mathrm{C}_{n m}=\frac{2 \hbar}{\pi^{5 / 2} \Lambda^{7}} \int_{0}^{\infty} 4 \pi P^{2} d P \int_{0}^{\infty} d p_{r} p_{r}^{3} \sum_{\sigma= \pm} \Lambda^{2} I_{n m}^{+\sigma}\left(z, a / \Lambda ; P, p_{r}\right) \equiv \frac{\hbar}{\Lambda^{5}} \tilde{\mathrm{C}}_{n m}\left(z, \frac{a}{\Lambda}\right), \\
I_{n m}^{+\sigma}\left(z, a / \Lambda ; P, p_{r}\right)=(2 \pi)^{2} \int_{-1}^{1} \int_{-1}^{1} d u d v \frac{d \tilde{\sigma}_{+\sigma}\left(p_{r}, a / \Lambda\right)}{d \Omega} F\left(z ; u, v, P, p_{r}\right) D_{n m}^{+\sigma}\left(u, v, P, p_{r}\right), \\
\frac{d \tilde{\sigma}_{\sigma \tau}}{d \Omega}=\Lambda^{-2} \frac{d \sigma_{\sigma \tau}}{d \Omega}, \quad D_{n m}^{+\sigma}=\left\langle\Delta_{+\sigma}\left(p^{n} \mathbf{p}\right) \cdot \Delta_{+\sigma}\left(p^{m} \mathbf{p}\right)\right\rangle,
\end{gathered}
$$

where we denote by brackets the angular average

$$
\langle\cdots\rangle \equiv \int \frac{d \varphi d \varphi^{\prime}}{(2 \pi)^{2}} \int d p_{r}^{\prime} \delta\left(p_{r}-p_{r}^{\prime}\right) \cdots
$$

and we defined dimensionless collision integral matrix elements and differential cross section, $\tilde{\mathrm{C}}_{n m}$ and $d \tilde{\sigma}_{\sigma \tau} / d \Omega$, respectively. We consider in this paper only spherically symmetric scattering cross sections that do not depend on the azimuthal angles. The angular integrations over $u, v$ can be evaluated analytically, and the integrals of $P$ and $p_{r}$ can be evaluated numerically.

In the high-temperature limit, $z=\rho \Lambda^{3} \ll 1$, the quantum statistical factors can be neglected, and the distribution function has the Maxwell-Boltzmann form. The collision integrals then read

$$
I_{n m}^{+\sigma}\left(z, a / \Lambda ; P, p_{r}\right) \rightarrow(2 \pi)^{2} \rho_{+} \rho_{\sigma} e^{-P^{2} / 2-2 p_{r}^{2}} \int_{-1}^{1} \int_{-1}^{1} d u d v \frac{d \tilde{\sigma}_{+\sigma}\left(p_{r}, a / \Lambda\right)}{d \Omega} D_{n m}^{+\sigma}\left(u, v, P, p_{r}\right)
$$

and can be expressed in terms of incomplete $\Gamma$ functions.

Similarly, for the computation of the spin-heat relaxation rate, we encounter the following integral:

$$
\begin{aligned}
\left\langle\left(\frac{\epsilon_{\mathbf{p}}}{k_{B} T}\right) \hat{C}_{s}\left(\frac{\epsilon_{\mathbf{p}}}{k_{B} T}\right)\right\rangle & =\frac{1}{2 \pi^{7 / 2} \Lambda^{5} \hbar} \int_{0}^{\infty} 4 \pi P^{2} d P \int_{0}^{\infty} d p_{r} p_{r}^{3} \Lambda^{2} I_{\mathrm{st}}^{+-}\left(z, a / \Lambda ; P, p_{r}\right) \equiv \frac{\tilde{\mathrm{C}}_{\mathrm{st}}(z, a / \Lambda)}{\Lambda^{3} \hbar}, \\
I_{\mathrm{st}}^{+-}\left(z, a / \Lambda ; P, p_{r}\right) & =(2 \pi)^{2} \int_{-1}^{1} \int_{-1}^{1} d u d v \frac{d \tilde{\sigma}_{+-}\left(p_{r}, a / \Lambda\right)}{d \Omega} F\left(z ; u, v, P, p_{r}\right) D_{\mathrm{st}}\left(P, p_{r}, u, v\right), \\
D_{\mathrm{st}}\left(P, p_{r}, u, v\right) & =\left\langle\Delta_{+-}\left(\epsilon_{\mathbf{p}}\right)^{2}\right\rangle=4 P^{2} p_{r}^{2}(v-u)^{2} .
\end{aligned}
$$

The angular factors are calculated in the next section. In three dimensions, the angular integrations can be done analytically. The simplest one is given by

$$
I_{00}^{+-}\left(z ; P, p_{r}\right)=\frac{d \tilde{\sigma}_{+\sigma}\left(p_{r}, a / \Lambda\right)}{d \Omega} \frac{512 \sqrt{\pi}}{\frac{2}{\pi^{5 / 2}} 4 \pi P^{2}} \frac{z^{2} e^{\frac{p^{2}}{2}+2 p_{r}^{2}}}{\left(e^{\frac{p^{2}}{2}+2 p_{r}^{2}}-z^{2}\right)^{2}} \ln \left[e^{-\frac{1}{2}\left(p_{1}^{2}-p_{2}^{2}\right)} \frac{e^{p_{1}^{2}}-z}{e^{p_{2}^{2}}-z}\right] \ln \left[e^{-\left(p_{1}^{2}-p_{2}^{2}\right)}\left(\frac{e^{p_{1}^{2}}-z}{e^{p_{2}^{2}}-z}\right)^{2}\right],
$$

where $p_{1,2}$ are to be expressed in terms of center-of-mass coordinates [see Eq. (B1)]. In previous work [29] on spin drag, this integrand was written in terms of susceptibilities as a function of momentum transfer. 


\section{Angular integrations}

We show some details of the computation of the angular integral (B4), which we reproduce here for convenience,

$$
\begin{aligned}
I_{n m}^{+\sigma}\left(z ; P, p_{r}\right)= & \int_{-1}^{1} \int_{-1}^{1}(2 \pi)^{2} d u d v F\left(z ; u, v, P, p_{r}\right) \\
& \times D_{n m}^{+\sigma}\left(u, v, P, p_{r}\right) .
\end{aligned}
$$

We first compute $D_{n m}^{+\sigma}\left(u, v, P, p_{r}\right)$. Recalling that energy and momentum conservation results in $\Delta_{++}(\mathbf{p})=0$ and $\Delta_{++}\left(\epsilon_{\mathbf{p}}\right)=0, I_{00}$ and $I_{01}$ depend only on interspin scattering, so we need to compute only the following:

$$
\begin{aligned}
& \left\langle\Delta_{+-}^{2}\left(\epsilon_{\mathbf{p}}\right)\right\rangle,\left\langle\Delta_{+-}^{2}(\mathbf{p})\right\rangle,\left\langle\Delta_{+-}(\mathbf{p}) \cdot \Delta_{+-}\left(p^{2} \mathbf{p}\right)\right\rangle, \\
& \left\langle\Delta_{+}^{2}\left(p^{2} \mathbf{p}\right)\right\rangle,\left\langle\Delta_{+-}^{2}\left(p^{2} \mathbf{p}\right)\right\rangle,
\end{aligned}
$$

where we recall $\langle\cdots\rangle \equiv \int \frac{d \varphi d \varphi^{\prime}}{(2 \pi)^{2}} \int d p_{r}^{\prime} \delta\left(p_{r}-p_{r}^{\prime}\right) \ldots$ Taking $p_{r}=p_{r}^{\prime}$ ahead of time, we find

$$
\begin{aligned}
\Delta_{+-}(\mathbf{p}) & =2 \mathbf{p}_{r}^{\prime}-2 \mathbf{p}_{r}, \quad \Delta_{+-}\left(\epsilon_{\mathbf{p}}\right)=2 \mathbf{P} \cdot\left(\mathbf{p}_{r}^{\prime}-\mathbf{p}_{r}\right), \\
\Delta_{++}\left(p^{2} \mathbf{p}\right) & =2\left(\mathbf{P} \cdot \mathbf{p}_{r}^{\prime}\right) \mathbf{p}_{r}^{\prime}-2\left(\mathbf{P} \cdot \mathbf{p}_{r}\right) \mathbf{p}_{r}, \\
\Delta_{+-}\left(p^{2} \mathbf{p}\right) & =\mathbf{P} \cdot\left(\mathbf{p}_{r}^{\prime}-\mathbf{p}_{r}\right) \mathbf{P}+\left(P^{2} / 2+2 p_{r}^{2}\right)\left(\mathbf{p}_{r}^{\prime}-\mathbf{p}_{r}\right) ;
\end{aligned}
$$

hence,

$$
\begin{aligned}
\Delta_{+-}^{2}(\mathbf{p})= & 8 p_{r}^{2}\left(1-\hat{\mathbf{p}}_{r} \cdot \hat{\mathbf{p}}_{r}^{\prime}\right) \\
\Delta_{+-}^{2}\left(\epsilon_{\mathbf{p}}\right)= & 4 P^{2} p_{r}^{2}(v-u)^{2} \\
\Delta_{+-}(\mathbf{p}) \cdot \Delta_{+-}\left(p^{2} \mathbf{p}\right)= & 2 P^{2} p_{r}^{2}(u-v)^{2} \\
& +2 p_{r}^{2}\left(P^{2}+4 p_{r}^{2}\right)\left(1-\hat{\mathbf{p}}_{r} \cdot \hat{\mathbf{p}}_{r}^{\prime}\right) \\
\Delta_{++}^{2}\left(p^{2} \mathbf{p}\right)= & P^{2} p_{r}^{4}\left[4\left(u^{2}+v^{2}\right)-8 u v \hat{\mathbf{p}}_{r} \cdot \hat{\mathbf{p}}_{r}^{\prime}\right], \\
\Delta_{+-}^{2}\left(p^{2} \mathbf{p}\right)= & P^{2} p_{r}^{2}\left(3 P^{2}+4 p_{r}^{2}\right)(u-v)^{2} \\
& +2 p_{r}^{2}\left(P^{2} / 2+2 p_{r}^{2}\right)^{2}\left(1-\hat{\mathbf{p}}_{r} \cdot \hat{\mathbf{p}}_{r}^{\prime}\right) .
\end{aligned}
$$

In three dimensions, the average over azimuthal angles can be performed using the identity

$$
\mathbf{p}_{r} \cdot \mathbf{p}_{r}^{\prime}=\sin \theta \sin \theta^{\prime} \cos \left(\varphi-\varphi^{\prime}\right)+\cos \theta \cos \theta^{\prime},
$$

and since $\left\langle\cos \left(\varphi-\varphi^{\prime}\right)\right\rangle=0$, we have $\left\langle\mathbf{p}_{r} \cdot \mathbf{p}_{r}^{\prime}\right\rangle=u v$; hence,

$$
\begin{aligned}
& D_{00}^{++}=\left\langle\Delta_{++}^{2}(\mathbf{p})\right\rangle=0, \\
& D_{00}^{+-}=\left\langle\Delta_{+-}^{2}(\mathbf{p})\right\rangle=8 p_{r}^{2}(1-u v), \\
& D_{\mathrm{st}}^{+-}=\left\langle\Delta_{+-}^{2}\left(\epsilon_{\mathbf{p}}\right)\right\rangle=4 P^{2} p_{r}^{2}(v-u)^{2}, \\
& D_{01}^{++}=0,
\end{aligned}
$$

$$
\begin{aligned}
D_{01}^{+-}= & \left\langle\Delta_{+-}(\mathbf{p}) \cdot \Delta_{+-}\left(p^{2} \mathbf{p}\right)\right\rangle \\
= & \left.2 p_{r}^{2}\left[P^{2}(u-v)^{2}+\left(P^{2}+4 p_{r}^{2}\right)(1-u v)\right)\right], \\
D_{11}^{++}= & \left\langle\Delta_{++}^{2}\left(p^{2} \mathbf{p}\right)\right\rangle=P^{2} p_{r}^{4}\left[4\left(u^{2}+v^{2}\right)-8(u v)^{2}\right], \\
D_{11}^{+-}= & \left\langle\Delta_{+-}^{2}\left(p^{2} \mathbf{p}\right)\right\rangle=p_{r}^{2}\left[P^{2}\left(3 P^{2}+4 p_{r}^{2}\right)(u-v)^{2}\right. \\
& \left.+2\left(P^{2} / 2+2 p_{r}^{2}\right)^{2}(1-u v)\right] .
\end{aligned}
$$

\section{APPENDIX C: COMPARISON WITH SPIN-DRAG RELAXATION TIME}

In this section, we show that the leading term in the solution given in Sec. V is consistent with the spin-drag relaxation time $\tau_{\text {sd }}$, defined by $\sigma_{s}=\rho \tau_{\text {sd }} / m$, which has been computed in the literature in the absence of spin-heat currents [28,29]. For this purpose, we write the expansion coefficients as

$$
\left(\begin{array}{l}
c_{0}^{(F)} \\
c_{1}^{(F)}
\end{array}\right)=\frac{3 \rho}{\left(1-\mathrm{C}_{01}^{2} / \mathrm{C}_{00} \mathrm{C}_{11}\right)}\left(\begin{array}{l}
\frac{1}{\mathrm{C}_{00}}-\frac{w}{k_{B} T} \frac{\mathrm{C}_{01}}{\mathrm{C}_{00} \mathrm{C}_{11}} \\
\frac{w}{k_{B} T \mathrm{C}_{11}}-\frac{\mathrm{C}_{01}}{\mathrm{C}_{00} \mathrm{C}_{11}}
\end{array}\right) .
$$

From power counting, $\mathrm{C}_{n m} \propto \int d p p^{7+2 n+2 m}$, we expect that $\mathrm{C}_{00}<\mathrm{C}_{01}<\mathrm{C}_{11}$; hence, $\mathrm{C}_{01} / \mathrm{C}_{00} \mathrm{C}_{11} \ll 1$ and $\mathrm{C}_{00} / \mathrm{C}_{11} \ll 1$. Thus, to leading order in the ratios $\mathrm{C}_{01} / \mathrm{C}_{00} \mathrm{C}_{11}$ and $\mathrm{C}_{00} / \mathrm{C}_{11}$, the spin conductivity is given by

$$
\sigma_{s}=L_{F F}=\rho c_{0}^{(F)}=\frac{3 \rho^{2}}{\mathrm{C}_{00}},
$$

for which

$$
\frac{1}{\tau_{\mathrm{sd}}}=\frac{1}{m c_{0}^{(F)}}=\frac{\mathrm{C}_{00}}{3 m \rho}=\frac{\left\langle\mathbf{v}_{\mathbf{p}} \otimes \hat{C}_{s} \mathbf{v}_{\mathbf{p}}\right\rangle}{\left\langle\mathbf{v}_{\mathbf{p}} \otimes \mathbf{v}_{\mathbf{p}}\right\rangle} .
$$

This expression in terms of the inner product is consistent with that of Refs. [10,46].

This leading-order solution, given by $\phi_{\mathbf{p} s}=\tau_{\mathrm{sd}} \mathbf{v}_{p} \cdot \mathbf{F}_{s}$, describes a uniform shift of the equilibrium distributions of the spin-up and -down particles in opposite directions, resulting in a spin current. To this order, the spin conductivity is determined by the viscosity between up and down atoms that arises from interspin scattering, hence the name spin drag [47]. In contrast, the spin-heat conductivity, which depends on intraspin scattering, is finite even in the absence of interspin scattering.

The second-order solution which we have included in this paper represents a distortion of the local distribution and is necessary to capture coupled spin and heat flows because the energy current carried by the leading-order solution is subtracted in the definition of the heat current [see Eq. (11)].
[1] G. E. W. Bauer, E. Saitoh, and B. J. van Wees, Nat. Mater. 11, 391 (2012).

[2] J.-P. Brantut, C. Grenier, J. Meineke, D. Stadler, S. Krinner, C. Kollath, T. Esslinger, and A. Georges, Science 342, 713 (2013).

[3] C. Grenier, C. Kollath, and A. Georges, arXiv:1209.3942.

[4] A. Rancon, C. Chin, and K. Levin, New J. Phys. 16, 113072 (2014).

[5] E. L. Hazlett, L.-C. Ha, and C. Chin, arXiv:1306.4018.
[6] M. Hatami, G. E. W. Bauer, S. Takahashi, and S. Maekawa, Solid State Commun. 150, 480 (2010).

[7] T. S. Nunner and F. von Oppen, Phys. Rev. B 84, 020405 (2011).

[8] For bosons, the pseudospin refers to a sufficiently isolated subset of the atomic hyperfine states, taken from, for example, the ground-state manifold of ${ }^{87} \mathrm{Rb}$ discussed in Ref. [48].

[9] L. Vichi and S. Stringari, Phys. Rev. A 60, 4734 (1999).

[10] A. Sommer, M. Ku, G. Roati, and M. W. Zwierlein, Nature (London) 472, 201 (2011). 
[11] S. B. Koller, A. Groot, P. C. Bons, R. A. Duine, H. T. C. Stoof, and P. van der Straten, arXiv:1204.6143.

[12] M. Polini and G. Vignale, Phys. Rev. Lett. 98266403 (2007).

[13] C. P. Weber, N. Gedik, J. E. Moore, J. Orenstein, J. Stephens, and D. D. Awschalom, Nature (London) 437, 1330 (2005).

[14] T. Ohde, M. Bonitz, T. Bornath, D. Kremp, and M. Schlanges, Phys. Plasmas 3, 1241 (1996).

[15] M. Sultan, U. Atxitia, A. Melnikov, O. Chubykalo-Fesenko, and U. Bovensiepen, Phys. Rev. B 85, 184407 (2012).

[16] F. K. Dejene, J. Flipse, G. E. W. Bauer, and B. J. van Wees, Nat. Phys. 9, 636 (2013).

[17] I. J. Vera-Marun, B. J. van Wees, and R. Jansen, Phys. Rev. Lett. 112, 056602 (2014).

[18] Our work may also be relevant to the solid-state environment in regard to the contribution to the transport and relaxation coefficients coming from electron-electron interactions.

[19] A. A. Kovalev and Y. Tserkovnyak, Solid State Commun. 150, 500 (2010)

[20] C. H. Wong, H. J. van Driel, R. Kittinaradorn, H. T. C. Stoof, and R. A. Duine, Phys. Rev. Lett. 108, 075301 (2012).

[21] The $s$-wave interspin scattering is sufficient to stabilize the spin currents that we consider in this work. Intraspin relaxation for fermions due to $p$-wave scattering was considered in Ref. [37].

[22] L. D. Landau and E. M. Lifshitz, Fluid Mechanics (Pergamon, New York, 1987).

[23] For degenerate fermions in a solid-state environment, one usually subtracts only the chemical potential, which is related to the enthalpy by $w=\mu+T s$, but the difference is negligible because $s \sim T / T_{F}$.

[24] Such a relaxation term is also consistent with the theory of quasistationary thermodynamics [49], which assumes that for small deviations from equilibrium, a nonequilibrium quantity such as $T_{s}$ should decay as $\partial_{t} T_{s}=-T_{s} / \tau_{\mathrm{st}}$.

[25] We neglect here the temperature dependence of $\kappa_{s}$.

[26] We note that for fermions a superfluid transition occurs at $T_{c} \ll$ $T_{F}$ and that the results reported in this work do not include effects related to this transition. Depending on the value of $T_{c}$, the fermi gas may enter a Fermi-liquid regime for $T_{c}<T \ll T_{F}$. In this regime, our solution based on Boltzmann's equation remains valid provided that one uses Fermi-liquid scattering amplitudes in the collision integral as shown in Ref. [46].

[27] The measured values of the transport coefficients should be compared with the trap-averaged values, which differ slightly from the results presented here.

[28] R. A. Duine and H. T. C. Stoof, Phys. Rev. Lett. 103, 170401 (2009).
[29] H. J. van Driel, R. A. Duine, and H. T. C. Stoof, Phys. Rev. Lett. 105, 155301 (2010).

[30] H. Kim and D. A. Huse, Phys. Rev. A 86, 053607 (2012).

[31] P. Chaikin and T. Lubensky, Principles of Condensed Matter Physics (Cambridge University Press, Cambridge, 2000).

[32] S. Chapman and T. Cowling, The Mathematical Theory of Non-uniform Gases: An Account of the Kinetic Theory of Viscosity, Thermal Conduction and Diffusion in Gases, Cambridge Mathematical Library (Cambridge University Press, Cambridge, 1970).

[33] L. Pitaevski and E. M. Lifshitz, Physical Kinetics, 1st ed. (Pergamon, New York, 1981).

[34] In the center-of-mass frame, the reduced mass is $m / 2$.

[35] L. Reichl, A Modern Course in Statistical Physics, 2nd ed. (Wiley, New York, 1998).

[36] G. Mahan, in Good Thermoelectrics, Solid State Physics, Vol. 51 (Academic Press, London, 1997), pp. 81-157.

[37] C. H. Wong, H. T. C. Stoof, and R. A. Duine, Phys. Rev. A 85, 063613 (2012).

[38] In the high-temperature limit, it is more convenient instead to expand in terms of the Sonine polynomials, which are orthogonal $[32,33,50]$.

[39] Note that in the notation of Ref. [37], $a_{n}=c_{n}^{(F)}$ and $b_{n}=c_{n}^{(T)}$.

[40] It can be shown that using this method and truncating at a finite order always result in transport coefficients which are less than their exact values $[33,50]$.

[41] P. C. Hohenberg and B. I. Halperin, Rev. Mod. Phys. 49, 435 (1977)

[42] R. Pathria, Statistical Mechanics (Elsevier Science, Massachuesetts, 2011).

[43] We are not including the condensate density, which would be given by $\rho_{c}=z / 1-z$.

[44] We neglect spin correlations in this paper.

[45] This follows from the fact that the semiclassical distribution function is the Wigner transform of the density matrix. See, for example, Ref. [51].

[46] G. M. Bruun, New J. Phys. 13, 035005 (2011).

[47] However, high-order terms do involve intraspin scattering.

[48] Y. J. Lin, K. Jimenez-Garcia, and I. B. Spielman, Nature (London) 471, 83 (2011).

[49] L. D. Landau and E. M. Lifshitz, Statistical Physics, Part 1, 3rd ed. (Pergamon, New York, 1980).

[50] H. Smith and H. H. Jensen, Transport Phenomena (Oxford University Press, Oxford, 1989).

[51] M. Naraschewski and R. J. Glauber, Phys. Rev. A 59, 4595 (1999). 